\title{
A study of the maximal Abelian gauge in $S U(2)$ Euclidean Yang-Mills theory in the presence of the Gribov horizon
}

\author{
M.A.L. Capri ${ }^{*}$, V.E.R. Lemes $^{\dagger}$, R.F. Sobreiro ${ }^{\ddagger}$, \\ S.P. Sorella ${ }^{\S}$, R. Thibes $\|$ \\ UERJ, Universidade do Estado do Rio de Janeiro, \\ Rua São Francisco Xavier 524, 20550-013 Maracanã, \\ Rio de Janeiro, Brasil
}

\begin{abstract}
We pursue the study of $S U(2)$ Euclidean Yang-Mills theory in the maximal Abelian gauge by taking into account the effects of the Gribov horizon. The Gribov approximation, previously introduced in [1, is improved through the introduction of the horizon function, which is constructed under the requirements of localizability and renormalizability. By following Zwanziger's treatment of the horizon function in the Landau gauge, we prove that, when cast in local form, the horizon term of the maximal Abelian gauge leads to a quantized theory which enjoys multiplicative renormalizability, a feature which is established to all orders by means of the algebraic renormalization. Furthermore, it turns out that the horizon term is compatible with the local residual $U(1)$ Ward identity, typical of the maximal Abelian gauge, which is easily derived. As a consequence, the nonrenormalization theorem, $Z_{g} Z_{A}^{1 / 2}=1$, relating the renormalization factors of the gauge coupling constant $Z_{g}$ and of the diagonal gluon field $Z_{A}$, still holds in the presence of the Gribov horizon. Finally, we notice that a generalized dimension two gluon operator can be also introduced. It is BRST invariant on-shell, a property which ensures its multiplicative renormalizability. Its anomalous dimension is not an independent parameter of the theory, being obtained from the renormalization factors of the gauge coupling constant and of the diagonal antighost field.
\end{abstract}

*marcio@dft.if.uerj.br

${ }^{\dagger}$ vitor@dft.if.uerj.br

¥sobreiro@uerj.br

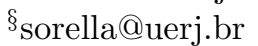

『Work supported by FAPERJ, Fundação de Amparo à Pesquisa do Estado do Rio de Janeiro, under the program Cientista do Nosso Estado, E-26/151.947/2004.

"thibes@dft.if.uerj.br 


\section{Introduction}

The maximal Abelian gauge [2, 3, 4] provides a useful tool in order to investigate nonperturbative aspects of Yang-Mills theories. This gauge turns out to be suitable for the study of the dual superconductivity mechanism for color confinement [5], according to which Yang-Mills theories in the low energy region should be described by an effective Abelian theory [6, 7, 8, 9] in the presence of monopoles. The condensation of these magnetic charges leads to a dual Meissner effect resulting in quark confinement. Here, the Abelian configurations are identified with the diagonal components $A_{\mu}^{i}, i=1, \ldots, N-1$, of the gauge field corresponding to the $(N-1)$ generators of the Cartan subgroup of $S U(N)$. The remaining off-diagonal components $A_{\mu}^{a}, a=1, \ldots, N^{2}-N$, corresponding to the $\left(N^{2}-N\right)$ off-diagonal generators of $S U(N)$, are expected to acquire a mass through a dynamical mechanism, thus decoupling at low energies.

The maximal Abelian gauge is a renormalizable gauge in the continuum [10, 11, 12, 13, while possessing a lattice formulation 3, 4. This property, shared only by few other gauges, such as the Landau and Coulomb ${ }^{1}$ gauges, gives to the maximal Abelian gauge a privileged role. It allows for a comparison between the results obtained in the continuum and in lattice numerical simulations.

Numerical studies of the behavior of the gluon propagator in the case of $S U(2)$ can be found in [15, 16]. In particular, according to [16], both diagonal and off-diagonal gluon propagators turn out to be suppressed in the infrared region. The diagonal propagator exhibits a Gribov like behavior, while the off-diagonal transverse component is of the Yukawa type. The value reported for the off-diagonal gluon mass is of approximately $1.2 \mathrm{GeV}$ [15, 16], and turns out to be twice bigger than the mass parameter entering the diagonal propagator. As a consequence, the off-diagonal propagator is short-ranged as compared to the diagonal one, a feature which is in agreement with the Abelian dominance hypothesis [6, 17, 8, 9].

As the Landau and Coulomb gauges, the maximal Abelian gauge is affected by the existence of the Gribov copies $[17]^{2}$, which have to be taken into account in order to properly quantize the theory. A first step in this direction was achieved in [1, where the original construction outlined by Gribov for the Landau gauge [17] was generalized to the maximal Abelian gauge. In particular, we have been able to generalize to the maximal Abelian gauge Gribov's result stating that for any field close to a horizon there is a gauge copy, close to the same horizon, located on the other side of the horizon [17. This result has provided support for restricting the domain of integration in the Feynman path integral to the so-called Gribov region, i.e. to the region in field space whose boundary is the first Gribov horizon, where the first vanishing eigenvalue of the Faddeev-Popov operator appears.

According to Gribov's original procedure [17, this restriction can be implemented by means of a no-pole condition on the ghost two-point function, which can be worked out order by order [17. In particular, the so called Gribov approximation amounts to work out the no-pole condition at the first nontrivial order, resulting in a new nonlocal term which has to be added to the Yang-Mills

\footnotetext{
${ }^{1}$ In the case of the Coulomb gauge we should remind that the issue of the renormalizability is still under debate, see 14] for a detailed account.

${ }^{2}$ See [18 for the construction of an explicit example of a zero mode of the Faddeev-Popov operator.
} 
action. In the Landau gauge, this term reads [17]

$$
S_{G}=-g^{2} \gamma^{4} N \int d^{4} x A_{\mu}^{A} \frac{1}{\partial^{2}} A_{\mu}^{A}
$$

where the index $A$ belongs to the adjoint representation of $S U(N), A=1, \ldots, N^{2}-1$, and where $\gamma$ is the Gribov parameter [17. It is not a free parameter of the theory, being defined by a gap equation which enables us to express it in terms of the gauge coupling $g$. At the first order, the gap equation defining $\gamma$ is given by

$$
1=\frac{3}{4} N g^{2} \int \frac{d^{4} k}{(2 \pi)^{4}} \frac{1}{k^{4}+2 N g^{2} \gamma^{4}} .
$$

Although expression (11), being quadratic in the gauge fields $A_{\mu}^{A}$, is useful to analyse the modifications of the tree level gluon propagator due to the restriction to the Gribov region, it suffers from several limitations. For instance, it does not allow to go beyond the tree level approximation, by taking into account in a consistent way the effects of the higher order quantum corrections, which would provide a better comparison with the results obtained from lattice numerical simulations as well as from the studies of the Schwinger-Dyson equations. The resolution of this important issue is due to Zwanziger who, by a recursive characterization of the lowest eigenvalue of the Faddeev-Popov operator, $-\partial_{\mu} D_{\mu}^{A B}=-\partial_{\mu}\left(\partial_{\mu} \delta^{A B}+g f^{A C B} A_{\mu}^{C}\right)$, has obtained a closed expression for the nonlocal term which implements the restriction to the Gribov region in the Landau gauge [19, 20]. This term, known as the horizon function, is given by

$$
S_{Z W}=-g^{2} \gamma^{4} \int d^{4} x f^{A B C} A_{\mu}^{B}\left[\left(\partial_{\mu} D_{\mu}\right)^{-1}\right]^{A D} f^{D E C} A_{\mu}^{E}
$$

Notice that Zwanziger's horizon term, eq.(3), reduces to the Gribov term (11) in the quadratic approximation, in which the Faddeev-Popov operator, $-\partial_{\mu} D_{\mu}^{A B}$, is replaced by the Laplacian, i.e. by $-\delta^{A B} \partial^{2}$. To some extent, Zwanziger's solution amounts to perform a re-summation of all higher order terms neglected in Gribov's quadratic approximation. In spite of its apparent nonlocality, the horizon function, eq.(33), can be cast in local form through the introduction of a suitable set of additional fields. Remarkably, the resulting local action turns out to be multiplicatively renormalizable to all orders [19, 20, 21. This property has made possible to evaluate the higher orders quantum corrections by taking into account the effects of the restriction to the Gribov region. In 22] one finds a study at one loop order of the gluon condensate $\left\langle A_{\mu}^{A} A_{\mu}^{A}\right\rangle$ in the presence of the horizon term (3). More recently, the evaluation of the two loop quantum corrections to the gluon and ghost propagators and their relationship with the infrared freezing of the running coupling constant has been worked out in [23, 24. We emphasize that the restriction to the Gribov region, as implemented by the Zwanziger horizon function, gives us a useful theoretical framework in order to achieve a rather clear understanding of the origin of the infrared suppression of the gluon propagator as well as of the infrared enhancement of the ghost propagator observed in the Landau gauge in both lattice numerical simulations [25, 26, 27, 28, 29, 30, 31, 32, 33] and Schwinger-Dyson approach [34, 35, 36, 37, 38.

Concerning now the maximal Abelian gauge, till now, the restriction to the Gribov region has been implemented within Gribov's quadratic approximation, as reported in [1] in the case of $S U(2)$. 
For the analogue of the nonlocal term (11) we have obtained

$$
S_{G}^{M A G}=-2 \gamma^{4} g^{2} \int d^{4} x A_{\mu} \frac{1}{\partial^{2}} A_{\mu}
$$

where $A_{\mu}$ is the diagonal component of the gauge field, defined according to the decomposition

$$
\mathcal{A}_{\mu}=A_{\mu}^{a} T^{a}+A_{\mu} T^{3}
$$

where $T^{a}, a=1,2$, denote the off-diagonal generators of $S U(2)$, while $T^{3}$ stands for the diagonal generator,

$$
\begin{aligned}
& {\left[T^{a}, T^{b}\right]=i \varepsilon^{a b} T^{3},} \\
& {\left[T^{3}, T^{a}\right]=i \varepsilon^{a b} T^{b},}
\end{aligned}
$$

where

$$
\begin{aligned}
\varepsilon^{a b} & =\varepsilon^{a b 3}, \\
\varepsilon^{a b} \varepsilon^{c d} & =\delta^{a c} \delta^{b d}-\delta^{a d} \delta^{b c} .
\end{aligned}
$$

As in the case of the Landau gauge, the Gribov parameter $\gamma$ is not free, being determined [1] by a gap equation ${ }^{3}$ similar to eq.(2). The addition of the Gribov term (4) to the Yang-Mills action deeply modifies the infrared behavior of the gluon and ghost propagators in the maximal Abelian gauge. Let us mention here that the results which we have obtained [1] for the diagonal and offdiagonal components of the gluon propagator are in qualitative agreement with those reported in [16], see next section for a brief review. Nevertheless, the nonlocal term (4) suffers from the same limitations of the corresponding term, eq.(11), of the Landau gauge, requiring thus the construction of the analogue of Zwanziger's horizon term, eq.(3). This is the task of the present work.

Before starting the description of the technical aspects, it might be worth to give a short account of how the horizon term of the maximal Abelian gauge has been identified. Having constructed this term in the Gribov approximation, eq.(4), our strategy has been that of looking for a possible extension of it, which enjoys the properties of localizability and renormalizability. In other words, one looks first at a possible nonlocal term which reduces to eq.(4) in the quadratic approximation. Further, one requires that such nonlocal term can be cast in local form by means of the introduction of a suitable additional set of fields. Finally, the resulting local theory has to be multiplicatively renormalizable. We underline that the requirements of localizability and renormalizability are unavoidable in order to have at our disposal a consistent computational framework. These are strong requirements, which yield a very powerful criterion in order to deal with nonlocal terms ${ }^{4}$. In the present case, a unique solution for the horizon term in the maximal

\footnotetext{
${ }^{3}$ In the case of the maximal Abelian gauge, and for the gauge group $S U(2)$, the gap equation defining the Gribov parameter $\gamma$ reads [1]

$$
1=\frac{3}{4} g^{2} \int \frac{d^{4} k}{(2 \pi)^{4}} \frac{1}{k^{4}+\gamma^{4}} .
$$

${ }^{4}$ See for instance the recent results [39, 40] on the construction of a renormalizable nonabelian massive model based on the use of the gauge invariant nonlocal operator $\operatorname{Tr} \int d^{4} x F_{\mu \nu} \frac{1}{D^{2}} F_{\mu \nu}$.
} 
Abelian gauge, which reduces to expression (41) and which fulfills the requirements of localizability and renormalizability, has in fact emerged from our analysis. It reads

$$
S_{\text {Horizon }}=\gamma^{4} g^{2} \int d^{4} x \varepsilon^{a b} A_{\mu}\left(\mathcal{M}^{-1}\right)^{a c} \varepsilon^{c b} A_{\mu},
$$

where $\mathcal{M}^{a b}$ stands for the off-diagonal Faddeev-Popov operator of the maximal Abelian gauge

$$
\mathcal{M}^{a b}=-D_{\mu}^{a c} D_{\mu}^{c b}-g^{2} \varepsilon^{a c} \varepsilon^{b d} A_{\mu}^{c} A_{\mu}^{d},
$$

with $D_{\mu}^{a b}$ being the covariant derivative with respect to the diagonal component $A_{\mu}$,

$$
D_{\mu}^{a b}=\delta^{a b} \partial_{\mu}-g \varepsilon^{a b} A_{\mu} .
$$

Notice that the horizon term of eq.(8) reduces precisely to expression (44) in the quadratic approximation, amounting to replace the operator $\mathcal{M}^{a b}$ by the Laplacian, i.e. by $-\delta^{a b} \partial^{2}$. No other solution for the horizon term in the maximal Abelian gauge, fulfilling the requirements of localizability and renormalizability, has been found. Although several candidates which reduce to expression (44) can be easily written down, they are ruled out by the requirement of localizability and renormalizabilty. Examples of such terms can be obtained from (44) by including more spacetime derivatives in the numerator, while considering higher powers of the Faddeev-Popov operator $\mathcal{M}^{a b}$ in the denominator ${ }^{5}$. However, the localization procedure of such terms would require the introduction of dimensionless auxiliary fields, a feature which jeopardizes the renormalizability of the resulting action ${ }^{6}$. Expression (8) can be seen thus as the minimal extension of the nonlocal action (4). It shares great similarity with Zwanziger's horizon term in the Landau gauge, eq.(3), as expressed by the presence of the inverse of the Faddeev-Popov operator, $\left(\mathcal{M}^{-1}\right)^{a b}$, which looks rather natural. We remind in fact that the diagonal ghost sector turns out to be unaffected by the presence of the Gribov copies, see Appendix B of [1]. The proof of the localizability and of the multiplicative renormalizability of expression (8) constitutes our main result.

The present work is organized as follows. In Sect.2 we give a brief account of the construction of the maximal Abelian gauge fixing condition and of the results obtained in [1] on the infrared behavior of the gluon and ghost propagators. In Sect.3 we implement the localization procedure for the nonlocal horizon term (8) , presenting the $B R S T$ invariance as well as the rather rich set of additional global symmetries of the resulting local action. Sect.4 is devoted to a detailed proof of the multiplicative renormalizability of the theory. The proof extends to all orders by means of the use of the algebraic renormalization [42. An interesting feature of the theory is represented by the existence of a residual local $U(1)$ Ward identity, typical of the maximal Abelian gauge. As a consequence, the nonrenormalization theorem, $Z_{g} Z_{A}^{1 / 2}=1$, relating the renormalization factors

\footnotetext{
${ }^{5}$ An example of a term of this kind is given by the expression $\int d^{4} x \varepsilon^{a b} A_{\mu} \frac{\partial^{2}}{\mathcal{M}^{a d} \mathcal{M}^{d c}} \varepsilon^{c b} A_{\mu}$.

${ }^{6}$ Even if our present analysis has relied on a direct inspection of possible candidates, it is worth mentioning that the requirements of localizability and renormalizability might be linked in a natural way to the deformation technique introduced in 41, which could provide a more systematic way to look at the construction of the horizon term for a generic gauge, once the corresponding horizon function in the Gribov approximation has been established. Essentially, this would amount to start from the localized form of the horizon term in the Gribov approximation, and search for the most general local deformation which preserves all symmetries of the starting model as well as its field content. By means of the use of the master equation and of the antifield formalism, all possible deformations can be identified in terms of cohomolgy classes of the nilpotent functional operator associated to the master equation. The construction of the extension of Gribov's horizon term through the deformation approach is under investigation.
} 
of the gauge coupling constant $Z_{g}$ and of the diagonal gluon field $Z_{A}$, still holds in the presence of the Gribov horizon. In Sect.5 we introduce a generalized dimension two gluon operator and we prove its multiplicative renormalizability in the presence of the horizon term (8) . Furthermore, its anomalous dimension is not an independent parameter of the theory, being obtained from the renormalization factors of the gauge coupling constant $g$ and of the diagonal anti-ghost field. Sect.6 contains a few concluding remarks on further developments and on potential applications for lattice numerical simulations.

\section{The gauge fixing condition for the maximal Abelian gauge}

In this section we introduce the gauge fixing condition for the maximal Abelian gauge, providing a short account of the main results on the infrared behavior of the gluon and ghost propagators obtained in [1].

Similarly to the decomposition of the gauge field $\mathcal{A}_{\mu}$ given in expression (5), for the field strength one has

$$
\mathcal{F}_{\mu \nu}=F_{\mu \nu}^{a} T^{a}+F_{\mu \nu} T^{3},
$$

with the off-diagonal and diagonal parts given by

$$
\begin{aligned}
& F_{\mu \nu}^{a}=D_{\mu}^{a b} A_{\nu}^{b}-D_{\nu}^{a b} A_{\mu}^{b}, \\
& F_{\mu \nu}=\partial_{\mu} A_{\nu}-\partial_{\nu} A_{\mu}+g \varepsilon^{a b} A_{\mu}^{a} A_{\nu}^{b},
\end{aligned}
$$

where the covariant derivative $D_{\mu}^{a b}$ has been defined in eq.(10). For the Yang-Mills action in Euclidean space one obtains

$$
S_{\mathrm{YM}}=\frac{1}{4} \int d^{4} x\left(F_{\mu \nu}^{a} F_{\mu \nu}^{a}+F_{\mu \nu} F_{\mu \nu}\right)
$$

As it is easily checked, the classical action (13) is left invariant by the gauge transformations

$$
\begin{aligned}
\delta A_{\mu}^{a} & =-D_{\mu}^{a b} \omega^{b}-g \varepsilon^{a b} A_{\mu}^{b} \omega, \\
\delta A_{\mu} & =-\partial_{\mu} \omega-g \varepsilon^{a b} A_{\mu}^{a} \omega^{b} .
\end{aligned}
$$

The maximal Abelian gauge is obtained by demanding that the off-diagonal components $A_{\mu}^{a}$ of the gauge field obey the nonlinear condition

$$
D_{\mu}^{a b} A_{\mu}^{b}=0
$$

which follows by requiring that the auxiliary functional

$$
\mathcal{R}[A]=\int d^{4} x A_{\mu}^{a} A_{\mu}^{a},
$$

is stationary with respect to the gauge transformations (14). Moreover, as it is apparent from the presence of the covariant derivative $D_{\mu}^{a b}$, equation (15) allows for a residual local $U(1)$ invariance corresponding to the diagonal subgroup of $S U(2)$. This additional invariance has to be fixed by 
means of a suitable gauge condition on the diagonal component $A_{\mu}$, which will be chosen to be of the Landau type, also adopted in lattice simulations, namely

$$
\partial_{\mu} A_{\mu}=0 \text {. }
$$

The Faddeev-Popov operator, $\mathcal{M}^{a b}$, corresponding to the gauge condition (15) is easily derived by taking the second variation of the auxiliary functional $\mathcal{R}[A]$, being given by

$$
\mathcal{M}^{a b}=-D_{\mu}^{a c} D_{\mu}^{c b}-g^{2} \varepsilon^{a c} \varepsilon^{b d} A_{\mu}^{c} A_{\mu}^{d} .
$$

It enjoys the property of being Hermitian and, as pointed out in [18, is the difference of two positive semidefinite operators given, respectively, by $-D_{\mu}^{a c} D_{\mu}^{c b}$ and $g^{2} \varepsilon^{a c} \varepsilon^{b d} A_{\mu}^{c} A_{\mu}^{d}$.

As discussed in [1, in order to deal with the existence of Gribov copies which affect the gauge condition (15), one proceeds by restricting the domain of integration in the Feynman path integral to the so called Gribov region $\mathcal{C}_{0}$, defined as the set of fields fulfilling the gauge conditions (15), (17) and for which the Faddeev-Popov operator $\mathcal{M}^{a b}$ is positive definite, namely

$$
\mathcal{C}_{0}=\left\{A_{\mu}, A_{\mu}^{a}, \partial_{\mu} A_{\mu}=0, D_{\mu}^{a b} A_{\mu}^{b}=0, \mathcal{M}^{a b}=-D_{\mu}^{a c} D_{\mu}^{c b}-g^{2} \varepsilon^{a c} \varepsilon^{b d} A_{\mu}^{c} A_{\mu}^{d}>0\right\} .
$$

The boundary, $\partial \mathcal{C}_{0}$, of the region $\mathcal{C}_{0}$, where the first vanishing eigenvalue of $\mathcal{M}^{a b}$ appears, is called the first Gribov horizon. The restriction of the domain of integration to this region is supported by the possibility of generalizing to the maximal Abelian gauge [1] Gribov's original result [17] stating that for any field located near a horizon there is a gauge copy, close to the same horizon, located on the other side of the horizon.

The restriction to the region $\mathcal{C}_{0}$ can be implemented through the no-pole condition on the offdiagonal two point ghost function. To the first order, this condition amounts to the introduction of the nonlocal term given in expression (41). As a consequence, the tree level gluon and ghost propagators get deeply modified in the infrared. More precisely, both off-diagonal and diagonal transverse components of the gluon propagator turn out to be suppressed in the infrared [1]. The diagonal component of the gluon propagator is found to display the characteristic Gribov type behavior, i.e.

$$
\left\langle A_{\mu}(k) A_{\nu}(-k)\right\rangle=\frac{k^{2}}{k^{4}+\gamma^{4}}\left(\delta_{\mu \nu}-\frac{k_{\mu} k_{\nu}}{k^{2}}\right) .
$$

The off-diagonal propagator turns out to be of the Yukawa type, being given by

$$
\begin{aligned}
\left\langle A_{\mu}^{a}(k) A_{\nu}^{b}(-k)\right\rangle & =\delta^{a b} \frac{1}{k^{2}+m^{2}}\left(\delta_{\mu \nu}-\frac{k_{\mu} k_{\nu}}{k^{2}}\right), \\
a, b & =1,2
\end{aligned}
$$

where $m$ denotes the off-diagonal dynamical mass originating from the dimension two gluon condensate $\left\langle A_{\mu}^{a} A_{\mu}^{a}\right\rangle$ [12, 1]. As already remarked, the behavior of the transverse diagonal and offdiagonal gluon propagators, eqs.(20), (21), is in qualitative agreement with the lattice results [16]. In the case of the ghost propagator, it turns out that the off-diagonal component exhibits infrared enhancement, according to

$$
\begin{aligned}
\left.\mathcal{G}(k)\right|_{k=0} & \approx \frac{\gamma^{2}}{k^{4}} \\
\mathcal{G}(k) & =\frac{1}{2} \sum_{a}\left\langle\bar{c}^{a}(k) c^{a}(-k)\right\rangle,
\end{aligned}
$$


where $\left(\bar{c}^{a}, c^{a}\right)$ stand for the off-diagonal Faddeev-Popov ghosts. Also, the diagonal component of the ghost propagator turns out to be not affected by the restriction to the first horizon.

\section{Localization of the horizon function and symmetry con- tent}

\subsection{Local action from the horizon}

In this section we describe the localization procedure for the horizon term. Let us start by considering the partition function of Yang-Mills theory quantized in the maximal Abelian gauge, in the presence of the horizon term of eq.(8), namely

$$
\mathcal{Z}=\int D A^{a} D A D b^{a} D b D \bar{c}^{a} D c^{a} D \bar{c} D c \exp \left(-\left(S_{\mathrm{YM}}+S_{\mathrm{MAG}}+S_{\text {Horizon }}\right)\right)
$$

where $S_{\text {YM }}$ stands for the Yang-Mills action, eq.(13), and $S_{\mathrm{MAG}}$ denotes the gauge fixing term corresponding to the gauge conditions of eqs.(15),(17). The fields $\left(c^{a}, \bar{c}^{a}\right)$ are the off-diagonal ghosts and antighosts, while $(c, \bar{c})$ denote the diagonal ghost and antighost. Also, $\left(b^{a}, b\right)$ are the off-diagonal and diagonal Lagrange multipliers enforcing conditions (15) and (17). $S_{\mathrm{MAG}}$ is given by the following expression [11, 12]

$$
S_{\mathrm{MAG}}=\int d^{4} x\left(b^{a} D_{\mu}^{a b} A_{\mu}^{b}-\bar{c}^{a} \mathcal{M}^{a b} c^{b}+g \varepsilon^{a b} \bar{c}^{a} c D_{\mu}^{b c} A_{\mu}^{c}+b \partial_{\mu} A_{\mu}+\bar{c} \partial_{\mu}\left(\partial_{\mu} c+g \varepsilon^{a b} A_{\mu}^{a} c^{b}\right)\right),
$$

and $S_{\text {Horizon }}$ is the horizon term (8)

$$
S_{\text {Horizon }}=\gamma^{4} g^{2} \int d^{4} x \varepsilon^{a b} A_{\mu}\left(\mathcal{M}^{-1}\right)^{a c} \varepsilon^{c b} A_{\mu} .
$$

As in the case of the Landau gauge [19, 20], the horizon function (25) can be localized by means of a pair of complex vector bosonic fields, $\left(\phi_{\mu}^{a b}, \bar{\phi}_{\mu}^{a b}\right), a, b=1,2$, according to

$$
e^{-S_{\text {Horizon }}}=\int D \bar{\phi} D \phi(\operatorname{det} \mathcal{M})^{8} \exp \left(-\int d^{4} x\left(\bar{\phi}_{\mu}^{a b} \mathcal{M}^{a c} \phi_{\mu}^{c b}+\gamma^{2} g \varepsilon^{a b}\left(\phi_{\mu}^{a b}-\bar{\phi}_{\mu}^{a b}\right) A_{\mu}\right)\right)
$$

where the determinant, $(\operatorname{det} \mathcal{M})^{8}$, takes into account the Jacobian arising from the integration over the fields $\left(\phi_{\mu}^{a b}, \bar{\phi}_{\mu}^{a b}\right)$. This term can also be localized by means of a pair of vector anticommuting fields $\left(\omega_{\mu}^{a b}, \bar{\omega}_{\mu}^{a b}\right)$, namely

$$
(\operatorname{det} \mathcal{M})^{8}=\int D \bar{\omega} D \omega \exp \left(\int d^{4} x \bar{\omega}_{\mu}^{a b} \mathcal{M}^{a c} \omega_{\mu}^{c b}\right)
$$

Therefore, we obtain a local action which reads

$$
\begin{aligned}
\mathcal{Z} & =\int D \Psi e^{-S_{\text {Local }}}, \\
D \Psi & \equiv D A^{a} D A D b^{a} D b D \bar{c}^{a} D c^{a} D \bar{c} D c D \bar{\phi} D \phi D \bar{\omega} D \omega
\end{aligned}
$$


where

$$
S_{\mathrm{Local}}=S_{\mathrm{YM}}+S_{\mathrm{MAG}}+S_{\phi \omega}+S_{\gamma},
$$

with $S_{\phi \omega}, S_{\gamma}$ given by

$$
\begin{aligned}
S_{\phi \omega} & =\int d^{4} x\left(\bar{\phi}_{\mu}^{a b} \mathcal{M}^{a c} \phi_{\mu}^{c b}-\bar{\omega}_{\mu}^{a b} \mathcal{M}^{a c} \omega_{\mu}^{c b}\right) \\
S_{\gamma} & =\gamma^{2} g \int d^{4} x \varepsilon^{a b}\left(\phi_{\mu}^{a b}-\bar{\phi}_{\mu}^{a b}\right) A_{\mu} .
\end{aligned}
$$

\subsection{BRST invariance}

In order to establish the BRST invariance of the resulting local theory, we proceed as in [19, 20] and consider first the particular case when $\gamma=0$, i.e.

$$
\left.S_{\text {Local }}\right|_{\gamma=0}=S_{\mathrm{YM}}+S_{\mathrm{MAG}}+S_{\phi \omega} .
$$

In this case we have in fact introduced nothing more than a unity written as

$$
\int D \phi D \bar{\phi} D \omega D \bar{\omega} \exp \left(-\int d^{4} x\left(\bar{\phi}_{\mu}^{a b} \mathcal{M}^{a c} \phi_{\mu}^{c b}-\bar{\omega}_{\mu}^{a b} \mathcal{M}^{a c} \omega_{\mu}^{c b}\right)\right)=1 .
$$

Furthermore, the action (31) may be written in a BRST invariant fashion. To see this, let us introduce the following nilpotent $B R S T$ transformations

$$
\begin{aligned}
s A_{\mu}^{a} & =-\left(D_{\mu}^{a b} c^{b}+g \varepsilon^{a b} A_{\mu}^{b} c\right), \\
s A_{\mu} & =-\left(\partial_{\mu} c+g \varepsilon^{a b} A_{\mu}^{a} c^{b}\right), \\
s c^{a} & =g \varepsilon^{a b} c^{b} c, \\
s c & =\frac{g}{2} \varepsilon^{a b} c^{a} c^{b}, \\
s \bar{c}^{a} & =b^{a}, \quad s b^{a}=0 \\
s \bar{c} & =b, \quad s b=0, \\
s \phi_{\mu}^{a b} & =\omega_{\mu}^{a b}, \quad s \omega_{\mu}^{a b}=0 \\
s \bar{\omega}_{\mu}^{a b}=\bar{\phi}_{\mu}^{a b}, \quad s \bar{\phi}_{\mu}^{a b}=0 & \\
& s^{2}=0 .
\end{aligned}
$$

Now, let $S_{0}$ be the action defined by

$$
S_{0}=S_{\mathrm{YM}}+s \int d^{4} x\left(\bar{c}^{a} D_{\mu}^{a b} A_{\mu}^{b}+\bar{c} \partial_{\mu} A_{\mu}+\bar{\omega}_{\mu}^{a b} \mathcal{M}^{a c} \phi_{\mu}^{c b}\right),
$$

which satisfies

$$
s S_{0}=0 .
$$

Acting with the $B R S T$ operator $s$, and recalling the expression of the Faddeev-Popov operator $\mathcal{M}^{a b}$, eq.(18), we obtain

$$
S_{0}=S_{\mathrm{YM}}+S_{\mathrm{MAG}}+S_{\phi \omega}+\int d^{4} x \bar{\omega}_{\mu}^{a b} \mathcal{F}^{a c} \phi_{\mu}^{c b},
$$


with

$$
\begin{aligned}
\mathcal{F}^{a b}= & 2 g \varepsilon^{a c}\left(\partial_{\mu} c+g \varepsilon^{d e} A_{\mu}^{d} c^{e}\right) D_{\mu}^{c b}+g \varepsilon^{a b} \partial_{\mu}\left(\partial_{\mu} c+g \varepsilon^{c d} A_{\mu}^{c} c^{d}\right) \\
& -g^{2}\left(\varepsilon^{a c} \varepsilon^{b d}+\varepsilon^{a d} \varepsilon^{b c}\right) A_{\mu}^{d}\left(D_{\mu}^{c e} c^{e}+g \varepsilon^{c e} A_{\mu}^{e} c\right) .
\end{aligned}
$$

Expression (37) differs from the action (31) by the presence of the last term. However, following [19, 20], we may transform $\left.S_{\text {Local }}\right|_{\gamma=0}$ into $S_{0}$ by performing the following shift in the variable $\omega_{\mu}^{a b}$

$$
\omega_{\mu}^{a b} \rightarrow \omega_{\mu}^{a b}-\left(\mathcal{M}^{-1}\right)^{a c} \mathcal{F}^{c d} \phi_{\mu}^{d b}
$$

whose corresponding Jacobian turns out to be field independent. Thus, the following equivalence holds, namely

$$
\int D \Psi e^{-S_{0}}=\int D \Psi e^{-S_{\text {Local }} \gamma_{\gamma=0}}
$$

Further, let us consider the term $S_{\gamma}$ given by (301). One can easily check that the term $\varepsilon^{a b} A_{\mu} \bar{\phi}_{\mu}^{a b}$ which appears in $S_{\gamma}$ can be written as

$$
\varepsilon^{a b} A_{\mu} \bar{\phi}_{\mu}^{a b}=\varepsilon^{a b} s\left(A_{\mu} \bar{\omega}_{\mu}^{a b}\right)-\varepsilon^{a b}\left(\partial_{\mu} c+g \varepsilon^{c d} A_{\mu}^{c} c^{d}\right) \bar{\omega}_{\mu}^{a b} .
$$

Once again, according to [19, 20], we can eliminate the last term of eq. (41) by means of the change of variables

$$
\omega_{\mu}^{a b} \rightarrow \omega_{\mu}^{a b}-\left(\mathcal{M}^{-1}\right)^{a c} \gamma^{2} g \varepsilon^{c b}\left(\partial_{\mu} c+g \varepsilon^{d e} A_{\mu}^{d} c^{e}\right) .
$$

Therefore, for the partition function we obtain the final expression

$$
\mathcal{Z}=\int D \Psi e^{-\left[S_{0}+\gamma^{2} g \int d^{4} x \varepsilon^{a b}\left(A_{\mu} \phi_{\mu}^{a b}-s\left(A_{\mu} \bar{\omega}_{\mu}^{a b}\right)\right)\right]} .
$$

Notice also that, due to the identity

$$
\gamma^{2} g \int d^{4} x \varepsilon^{a b}\left(A_{\mu} \phi_{\mu}^{a b}-s\left(A_{\mu} \bar{\omega}_{\mu}^{a b}\right)\right)=\gamma^{2} \int d^{4} x\left(D_{\mu}^{a b} \phi_{\mu}^{b a}-s\left(D_{\mu}^{a b} \omega_{\mu}^{b a}\right)\right)
$$

expression (43) becomes

$$
\mathcal{Z}=\int D \Psi e^{-\left[S_{0}+\gamma^{2} \int d^{4} x\left(D_{\mu}^{a b} \phi_{\mu}^{b a}-s\left(D_{\mu}^{a b} \omega_{\mu}^{b a}\right)\right)\right]}
$$

Nevertheless, due to the term $D_{\mu}^{a b} \phi_{\mu}^{b a}$, the action

$$
S_{0}+\gamma^{2} \int d^{4} x\left(D_{\mu}^{a b} \phi_{\mu}^{b a}-s\left(D_{\mu}^{a b} \omega_{\mu}^{b a}\right)\right)
$$

is not yet $B R S T$ invariant, a point which can be dealt with by means of the introduction of a pair of BRST doublets of local external sources [19, 20], $\left(U_{\mu \nu}^{a b}, M_{\mu \nu}^{a b}\right)$ and $\left(V_{\mu \nu}^{a b}, N_{\mu \nu}^{a b}\right)$, which transform as

$$
\begin{array}{ll}
s U_{\mu \nu}^{a b}=-M_{\mu \nu}^{a b}, & s M_{\mu \nu}^{a b}=0, \\
s V_{\mu \nu}^{a b}=N_{\mu \nu}^{a b}, & s N_{\mu \nu}^{a b}=0 .
\end{array}
$$


As pointed out in [19, 20, the introduction of these external sources allows us to promote expression (46) to a $B R S T$ invariant action. In fact, let $S_{\text {sources }}$ be the action

$$
\begin{aligned}
S_{\text {sources }} & =s \int d^{4} x\left(-U_{\mu \nu}^{a c} D_{\mu}^{a b} \phi_{\nu}^{b c}+V_{\mu \nu}^{a c} D_{\mu}^{a b} \bar{\omega}_{\nu}^{b c}\right) \\
& =\int d^{4} x\left(M_{\mu \nu}^{a c} D_{\mu}^{a b} \phi_{\nu}^{b c}+U_{\mu \nu}^{a c} s\left(D_{\mu}^{a b} \phi_{\nu}^{b c}\right)+N_{\mu \nu}^{a c} D_{\mu}^{a b} \bar{\omega}_{\nu}^{b c}+V_{\mu \nu}^{a c} s\left(D_{\mu}^{a b} \bar{\omega}_{\nu}^{b c}\right)\right)
\end{aligned}
$$

which obviously satisfies

$$
s S_{\text {sources }}=0 .
$$

Moreover, when the sources $U, M, V, N$ attain their physical value [19, 20], defined by

$$
\begin{aligned}
\left.M_{\mu \nu}^{a b}\right|_{\mathrm{phys}} & =-\left.V_{\mu \nu}^{a b}\right|_{\mathrm{phys}}=\delta^{a b} \delta_{\mu \nu} \gamma^{2}, \\
\left.U_{\mu \nu}^{a b}\right|_{\mathrm{phys}} & =\left.N_{\mu \nu}^{a b}\right|_{\mathrm{phys}}=0,
\end{aligned}
$$

it immediately follows that

$$
\left.S_{\text {sources }}\right|_{\text {phys }}=\int d^{4} x\left(\gamma^{2} D_{\mu}^{a b} \phi_{\mu}^{b a}-\gamma^{2} s\left(D_{\mu}^{a b} \bar{\omega}_{\mu}^{b a}\right)\right)
$$

which is nothing but expression (46). One sees thus that the use of the external sources $U, M$, $V, N$ enables us to introduce an extended action $\Sigma_{0}$

$$
\Sigma_{0}=S_{0}+S_{\text {sources }}
$$

which enjoys the important property of being $B R S T$ invariant,

$$
s \Sigma_{0}=0
$$

while reducing to expression (46) when the sources attain their physical value, eq.(50). Explicitly, we have

$$
\begin{aligned}
\Sigma_{0}= & S_{Y M}+S_{\mathrm{MAG}}+s \int d^{4} x\left(\bar{\omega}_{\mu}^{a b} \mathcal{M}^{a c} \phi_{\mu}^{c b}-U_{\mu \nu}^{a c} D_{\mu}^{a b} \phi_{\nu}^{b c}+V_{\mu \nu}^{a c} D_{\mu}^{a b} \bar{\omega}_{\nu}^{b c}\right) \\
= & S_{\mathrm{YM}}+S_{\mathrm{MAG}}+\int d^{4} x\left(\bar{\phi}_{\mu}^{a b} \mathcal{M}^{a c} \phi_{\mu}^{c b}-\bar{\omega}_{\mu}^{a b} \mathcal{M}^{a c} \omega_{\mu}^{c b}+\bar{\omega}_{\mu}^{a b} \mathcal{F}^{a c} \phi_{\mu}^{c b}+M_{\mu \nu}^{a c} D_{\mu}^{a b} \phi_{\nu}^{b c}+N_{\mu \nu}^{a c} D_{\mu}^{a b} \bar{\omega}_{\nu}^{b c}\right. \\
& \left.+U_{\mu \nu}^{a c}\left[D_{\mu}^{a b} \omega_{\nu}^{b c}+g \varepsilon^{a b}\left(\partial_{\mu} c+g \varepsilon^{d e} A_{\mu}^{d} c^{e}\right) \phi_{\nu}^{b c}\right]+V_{\mu \nu}^{a c}\left[D_{\mu}^{a b} \bar{\phi}_{\nu}^{b c}+g \varepsilon^{a b}\left(\partial_{\mu} c+g \varepsilon^{d e} A_{\mu}^{d} c^{e}\right) \bar{\omega}_{\nu}^{b c}\right]\right) .
\end{aligned}
$$

\subsection{Inclusion of the quartic ghost term}

Although being $B R S T$ invariant, the action $\Sigma_{0}$ is not yet the most general classical action to start with. As discussed in previous works [11, 12, the nonlinearity of the gauge condition (15) requires the introduction of a quartic term in the Faddeev-Popov ghost fields

$$
\frac{\alpha}{2} g^{2} \bar{c}^{a} c^{a} \bar{c}^{b} c^{b}
$$


which is in fact needed for renormalizability purposes. The parameter $\alpha$ in expression (55) is a gauge parameter. In our case, due to the presence of the localizing fields $\left(\phi_{\mu}^{a b}, \bar{\phi}_{\mu}^{a b}, \omega_{\mu}^{a b}, \bar{\omega}_{\mu}^{a b}\right)$, the quartic ghost term (55) is introduced in a BRST invariant way through the following action $\Sigma_{\alpha}$

$$
\begin{aligned}
\Sigma_{\alpha}= & s \int d^{4} x \frac{\alpha}{2}\left(\bar{c}^{a} b^{a}-g \varepsilon^{a b} \bar{c}^{a} \bar{c}^{b} c+g^{2} \bar{\omega}_{\mu}^{a c} \phi_{\mu}^{a c}\left(\bar{\phi}_{\nu}^{b d} \phi_{\nu}^{b d}-\bar{\omega}_{\nu}^{b d} \omega_{\nu}^{b d}\right)-2 g^{2} \bar{\omega}_{\mu}^{a c} \phi_{\mu}^{a c} \bar{c}^{b} c^{b}\right) \\
= & \frac{\alpha}{2} \int d^{4} x\left(b^{a} b^{a}-2 g \varepsilon^{a b} b^{a} \bar{c}^{b} c+g^{2} \bar{c}^{a} c^{a} \bar{c}^{b} c^{b}+g^{2}\left(\bar{\phi}_{\mu}^{a c} \phi_{\mu}^{a c}-\bar{\omega}_{\mu}^{a c} \omega_{\mu}^{a c}\right)\left(\bar{\phi}_{\nu}^{b d} \phi_{\nu}^{b d}-\bar{\omega}_{\nu}^{b d} \omega_{\nu}^{b d}\right)\right. \\
& \left.-2 g^{2}\left(\bar{\phi}_{\mu}^{a c} \phi_{\mu}^{a c}-\bar{\omega}_{\mu}^{a c} \omega_{\mu}^{a c}\right) \bar{c}^{b} c^{b}+2 g^{2} \bar{\omega}_{\mu}^{a c} \phi_{\mu}^{a c} b^{b} c^{b}-2 g^{3} \bar{\omega}_{\mu}^{a d} \phi_{\mu}^{a d} \varepsilon^{b c} \bar{c}^{b} c^{c} c\right) .
\end{aligned}
$$

Adding the $B R S T$ invariant term $\Sigma_{\alpha}$ to the action $\Sigma_{0}$, modifies the equation of motion of the off-diagonal Lagrange multiplier $b^{a}$, according to

$$
\frac{\delta\left(\Sigma_{0}+\Sigma_{\alpha}\right)}{\delta b^{a}}=D_{\mu}^{a b} A_{\mu}^{b}+\alpha\left(b^{a}-g \varepsilon^{a b} \bar{c}^{b} c+g^{2} \bar{\omega}_{\mu}^{b c} \phi_{\mu}^{b c} c^{a}\right)
$$

Therefore, we see that maximal Abelian gauge condition (15), $D_{\mu}^{a b} A_{\mu}^{b}=0$, is attained in the limit $\alpha \rightarrow 0$, which has to be performed after the removal of the ultraviolet divergences [11, 12. We also remark that expression (56) contains a unique free parameter, namely, the gauge parameter $\alpha$. As a consequence, the whole term $\Sigma_{\alpha}$ vanishes in the limit $\alpha \rightarrow 0$, allowing us to integrate out the auxiliary fields $\left(\phi_{\mu}^{a b}, \bar{\phi}_{\mu}^{a b}, \omega_{\mu}^{a b}, \bar{\omega}_{\mu}^{a b}\right)$, thus recovering the horizon term (8) . As we shall see in the next section, this important feature follows from the fact that the action $\left(\Sigma_{0}+\Sigma_{\alpha}\right)$ fulfills several Ward identities which, in particular, uniquely fix the form of the term $\Sigma_{\alpha}$.

\subsection{The global $U(8)$ symmetry}

In addition to the $B R S T$ invariance, and in complete analogy with the case of the Landau gauge [19, 20], the action $\left(\Sigma_{0}+\Sigma_{\alpha}\right)$ displays a global symmetry $U(f), f=8$, expressed by

$$
\mathcal{Q}_{\mu \nu}^{a b}\left(\Sigma_{0}+\Sigma_{\alpha}\right)=0
$$

with

$$
\begin{aligned}
\mathcal{Q}_{\mu \nu}^{a b}= & \int d^{4} x\left(\phi_{\mu}^{c a} \frac{\delta}{\delta \phi_{\nu}^{c b}}-\bar{\phi}_{\nu}^{c b} \frac{\delta}{\delta \bar{\phi}_{\mu}^{c a}}+\omega_{\mu}^{c a} \frac{\delta}{\delta \omega_{\nu}^{c b}}-\bar{\omega}_{\nu}^{c b} \frac{\delta}{\delta \bar{\omega}_{\mu}^{c a}}\right. \\
& \left.+V_{\sigma \mu}^{c a} \frac{\delta}{\delta V_{\sigma \nu}^{c b}}-M_{\sigma \nu}^{c b} \frac{\delta}{\delta M_{\sigma \mu}^{c a}}+N_{\sigma \mu}^{c a} \frac{\delta}{\delta N_{\sigma \nu}^{c b}}-U_{\sigma \nu}^{c b} \frac{\delta}{\delta U_{\sigma \mu}^{c a}}\right) .
\end{aligned}
$$

The presence of the global invariance $U(8)$ means that one can make use [19, 20] of the composite index $i \equiv(a, \mu), i=1, \ldots, 8$. Therefore, setting

$$
\left(\phi_{\mu}^{a b}, \bar{\phi}_{\mu}^{a b}, \omega_{\mu}^{a b}, \bar{\omega}_{\mu}^{a b}\right)=\left(\phi_{i}^{a}, \bar{\phi}_{i}^{a}, \omega_{i}^{a}, \bar{\omega}_{i}^{a}\right)
$$

and

$$
\left(U_{\mu \nu}^{a b}, V_{\mu \nu}^{a b}, M_{\mu \nu}^{a b}, N_{\mu \nu}^{a b}\right)=\left(U_{\mu i}^{a}, V_{\mu i}^{a}, M_{\mu i}^{a}, N_{\mu i}^{a}\right)
$$




\begin{tabular}{lcccccccccccc}
\hline & $A$ & $b$ & $\bar{c}$ & $c$ & $\phi$ & $\bar{\phi}$ & $\omega$ & $\bar{\omega}$ & $U$ & $V$ & $M$ & $N$ \\
\hline \hline dimension & 1 & 2 & 2 & 0 & 1 & 1 & 1 & 1 & 2 & 2 & 2 & 2 \\
gh number & 0 & 0 & -1 & 1 & 0 & 0 & 1 & -1 & -1 & 0 & 0 & 1 \\
$\mathcal{Q}_{f}$-charge & 0 & 0 & 0 & 0 & 1 & -1 & 1 & -1 & -1 & 1 & -1 & 1 \\
\hline
\end{tabular}

Table 1: Quantum numbers of the fields and sources

we rewrite expression (52) as

$$
\begin{aligned}
\Sigma_{0}+\Sigma_{\alpha}= & S_{\mathrm{YM}}+S_{\mathrm{MAG}}+\int d^{4} x\left(\bar{\phi}_{i}^{a} \mathcal{M}^{a b} \phi_{i}^{b}-\bar{\omega}_{i}^{a} \mathcal{M}^{a b} \omega_{i}^{b}+\bar{\omega}_{i}^{a} \mathcal{F}^{a b} \phi_{i}^{b}+M_{\mu i}^{a} D_{\mu}^{a b} \phi_{i}^{b}+N_{\mu i}^{a} D_{\mu}^{a b} \bar{\omega}_{i}^{b}\right. \\
& \left.+U_{\mu i}^{a}\left[D_{\mu}^{a b} \omega_{i}^{b}+g \varepsilon^{a b}\left(\partial_{\mu} c+g \varepsilon^{c d} A_{\mu}^{c} c^{d}\right) \phi_{i}^{b}\right]+V_{\mu i}^{a}\left[D_{\mu}^{a b} \bar{\phi}_{i}^{b}+g \varepsilon^{a b}\left(\partial_{\mu} c+g \varepsilon^{c d} A_{\mu}^{c} c^{d}\right) \bar{\omega}_{i}^{b}\right]\right) \\
& +\frac{\alpha}{2} \int d^{4} x\left(b^{a} b^{a}-2 g \varepsilon^{a b} b^{a} \bar{c}^{b} c+g^{2} \bar{c}^{a} c^{a} \bar{c}^{b} c^{b}+g^{2}\left(\bar{\phi}_{i}^{a} \phi_{i}^{a}-\bar{\omega}_{i}^{a} \omega_{i}^{a}\right)\left(\bar{\phi}_{j}^{b} \phi_{j}^{b}-\bar{\omega}_{j}^{b} \omega_{j}^{b}\right)\right. \\
& \left.-2 g^{2}\left(\bar{\phi}_{i}^{a} \phi_{i}^{a}-\bar{\omega}_{i}^{a} \omega_{i}^{a}\right) \bar{c}^{b} c^{b}+2 g^{2} \bar{\omega}_{i}^{a} \phi_{i}^{a} b^{b} c^{b}-2 g^{3} \bar{\omega}_{i}^{a} \phi_{i}^{a} \varepsilon^{b c} \bar{c}^{b} c^{c} c\right)
\end{aligned}
$$

For the symmetry generator we have

$$
\begin{aligned}
\mathcal{Q}_{i j}= & \int d^{4} x\left(\phi_{i}^{a} \frac{\delta}{\delta \phi_{j}^{a}}-\bar{\phi}_{j}^{a} \frac{\delta}{\delta \bar{\phi}_{i}^{a}}+\omega_{i}^{a} \frac{\delta}{\delta \omega_{j}^{a}}-\bar{\omega}_{j}^{a} \frac{\delta}{\delta \bar{\omega}_{i}^{a}}\right. \\
& \left.+V_{\mu i}^{a} \frac{\delta}{\delta V_{\mu j}^{a}}-M_{\mu j}^{a} \frac{\delta}{\delta M_{\mu i}^{a}}+N_{\mu i}^{a} \frac{\delta}{\delta N_{\mu j}^{a}}-U_{\mu j}^{a} \frac{\delta}{\delta U_{\mu i}^{a}}\right) .
\end{aligned}
$$

By means of the trace of the operator $\mathcal{Q}_{i j}$, i.e., $\mathcal{Q}_{i i} \equiv \mathcal{Q}_{f}$, the $i$-valued fields turn out to possess an additional quantum number, displayed in Table (II), together with the dimension and the ghost number.

\section{Identification of the final complete classical action $\Sigma$}

Let us proceed by establishing the rich set of Ward identities which will enable us to analyse the renormalizability of the theory to all orders. Let us first identify the final complete action to start with. To this purpose, we have to properly define the composite field operators entering the $B R S T$ transformations (33), (47)). We notice that the BRST transformation of the gauge field $A_{\mu}^{a}$ can be written as the sum of two composite operators, i.e.

$$
s A_{\mu}^{a}=\mathcal{O}_{1}+\mathcal{O}_{2}
$$

where

$$
\mathcal{O}_{1}=-D_{\mu}^{a b} c^{b}, \quad \mathcal{O}_{2}=-g \varepsilon^{a b} A_{\mu}^{b} c .
$$

Moreover, thanks to the nilpotency of the $B R S T$ operator, $s^{2}=0$, it follows that

$$
s \mathcal{O}_{1}=-s \mathcal{O}_{2}
$$

Therefore, the two composite operators, $\mathcal{O}_{1}, \mathcal{O}_{2}$, can be defined by means of the introduction of the external sources $\left(\Omega_{\mu}^{a}, \tau_{\mu}^{a}, \xi_{\mu}^{a}\right)$

$$
\Sigma_{\mathrm{ext}}^{(1)}=\int d^{4} x\left(\Omega_{\mu}^{a}\left(-D_{\mu}^{a b} c^{b}\right)+\tau_{\mu}^{a}\left(-g \varepsilon^{a b} A_{\mu} c\right)+\xi_{\mu}^{a} s\left(-g \varepsilon^{a b} A_{\mu} c\right)\right) .
$$


To guarantee the $B R S T$ invariance of $\Sigma_{\text {ext }}^{(1)}$, we require that

$$
\begin{aligned}
s \xi_{\mu}^{a} & =-\left(\Omega_{\mu}^{a}-\tau_{\mu}^{a}\right) \\
s \Omega_{\mu}^{a} & =s \tau_{\mu}^{a}=0 .
\end{aligned}
$$

Moreover, it is easily checked that the new action

$$
\Sigma_{0}+\Sigma_{\alpha}+\Sigma_{\text {ext }}^{(1)}
$$

is left invariant by the following set of transformations:

- the $\delta_{i}$ symmetry

$$
\begin{aligned}
\delta_{i} \bar{c}^{a} & =\phi_{i}^{a}, \\
\delta_{i} \bar{\phi}_{j}^{a} & =\delta_{i j} c^{a}, \\
\delta_{i} b^{a} & =g \varepsilon^{a b} \phi_{i}^{b} c, \\
\delta_{i} \Omega_{\mu}^{a} & =V_{\mu i}^{a},
\end{aligned}
$$

- the $\tilde{\delta}_{i}$ symmetry

$$
\begin{aligned}
\tilde{\delta}_{i} \bar{c}^{a} & =\bar{\omega}_{i}^{a}, \\
\tilde{\delta}_{i} \omega_{j}^{a} & =-\delta_{i j} c^{a}, \\
\tilde{\delta}_{i} b^{a} & =g \varepsilon^{a b} \bar{\omega}_{i}^{b} c, \\
\tilde{\delta}_{i} \Omega_{\mu}^{a} & =-U_{\mu i}^{a},
\end{aligned}
$$

with

$$
\delta_{i}\left(\Sigma_{0}+\Sigma_{\alpha}+\Sigma_{\text {ext }}^{(1)}\right)=\tilde{\delta}_{i}\left(\Sigma_{0}+\Sigma_{\alpha}+\Sigma_{\text {ext }}^{(1)}\right)=0 .
$$

As transformations (70), (71) contain composite field operators, i.e. $g \varepsilon^{a b} \phi_{i}^{b} c$ and $g \varepsilon^{a b} \bar{\omega}_{i}^{b} c$, we define them by means of external sources $\left(\eta_{i}^{a}, \lambda_{i}^{a}\right)$ and $\left(\vartheta_{i}^{a}, \rho_{i}^{a}\right)$, giving rise to two set of $B R S T$ doublets

$$
\begin{array}{ll}
s \eta_{i}^{a}=\lambda_{i}^{a}, & s \lambda_{i}^{a}=0, \\
s \vartheta_{i}^{a}=\rho_{i}^{a}, & s \rho_{i}^{a}=0,
\end{array}
$$

so that

$$
\begin{aligned}
\Sigma_{\mathrm{ext}}^{(2)}= & s \int d^{4} x g \varepsilon^{a b}\left(\eta_{i}^{a} \phi_{i}^{b} c+\vartheta_{i}^{a} \bar{\omega}_{i}^{b} c\right) \\
= & \int d^{4} x\left(g \varepsilon^{a b} \lambda_{i}^{a} \phi_{i}^{b} c+\eta_{i}^{a}\left[g \varepsilon^{a b} \omega_{i}^{b} c+\frac{g^{2}}{2} \varepsilon^{a b} \varepsilon^{c d} \phi_{i}^{b} c^{c} c^{d}\right]\right. \\
& \left.+g \varepsilon^{a b} \rho_{i}^{a} \bar{\omega}_{i}^{b} c-\vartheta_{i}^{a}\left[g \varepsilon^{a b} \bar{\phi}_{i}^{b} c-\frac{g^{2}}{2} \varepsilon^{a b} \varepsilon^{c d} \bar{\omega}_{i}^{b} c^{c} c^{d}\right]\right) .
\end{aligned}
$$


Further, introducing the $B R S T$ invariant sources $\left(\Omega_{\mu}, L^{a}, L\right)$, coupled respectively to the nonlinear $B R S T$ transformations of the fields $A_{\mu}, c^{a}, c$, for the final expression of the complete action $\Sigma$ we shall start with, we have

$$
\Sigma=\Sigma_{0}+\Sigma_{\alpha}+\Sigma_{\mathrm{ext}}^{(1)}+\Sigma_{\mathrm{ext}}^{(2)}+s \int d^{4} x\left(-\Omega_{\mu} A_{\mu}+L^{a} c^{a}+L c-\chi U_{\mu i}^{a} V_{\mu i}^{a}\right)
$$

namely

$$
\begin{aligned}
\Sigma= & S_{\mathrm{YM}}+S_{\mathrm{MAG}}+s \int d^{4} x\left(\bar{\omega}_{i}^{a} \mathcal{M}^{a b} \phi_{i}^{b}-U_{\mu i}^{a} D_{\mu}^{a b} \phi_{i}^{b}+V_{\mu i}^{a} D_{\mu}^{a b} \bar{\omega}_{i}^{b}+g \varepsilon^{a b} \eta_{i}^{a} \phi_{i}^{b} c+g \varepsilon^{a b} \vartheta_{i}^{a} \bar{\omega}_{i}^{b} c\right. \\
& \left.-\Omega_{\mu}^{a} A_{\mu}^{a}-g \varepsilon^{a b} \xi_{\mu}^{a} A_{\mu}^{b} c-\Omega_{\mu} A_{\mu}+L^{a} c^{a}+L c-\chi U_{\mu i}^{a} V_{\mu i}^{a}\right) \\
& +s \int d^{4} x \frac{\alpha}{2}\left(\bar{c}^{a} b^{a}-g \varepsilon^{a b} \bar{c}^{a} \bar{c}^{b} c+g^{2} \bar{\omega}_{i}^{a} \phi_{i}^{a}\left(\bar{\phi}_{j}^{b} \phi_{j}^{b}-\bar{\omega}_{j}^{b} \omega_{j}^{b}\right)-2 g^{2} \bar{\omega}_{i}^{a} \phi_{i}^{a} \bar{c}^{b} c^{b}\right) \\
= & S_{\mathrm{YM}}+\int d^{4} x\left(b^{a} D_{\mu}^{a b} A_{\mu}^{b}-\bar{c}^{a} \mathcal{M}^{a b} c^{b}+g \varepsilon^{a b} \bar{c}^{a} c D_{\mu}^{b c} A_{\mu}^{c}+b \partial_{\mu} A_{\mu}+\bar{c} \partial_{\mu}\left(\partial_{\mu} c+g \varepsilon^{a b} A_{\mu}^{a} c^{b}\right)\right. \\
& +\bar{\phi}_{i}^{a} \mathcal{M}^{a b} \phi_{i}^{b}-\bar{\omega}_{i}^{a} \mathcal{M}^{a b} \omega_{i}^{b}+\bar{\omega}_{i}^{a} \mathcal{F}^{a b} \phi_{i}^{b}+M_{\mu i}^{a} D_{\mu}^{a b} \phi_{i}^{b}+U_{\mu i}^{a}\left[D_{\mu}^{a b} \omega_{i}^{b}+g \varepsilon^{a b}\left(\partial_{\mu} c+g \varepsilon^{c d} A_{\mu}^{c} c^{d}\right) \phi_{i}^{b}\right] \\
& +N_{\mu i}^{a} D_{\mu}^{a b} \bar{\omega}_{i}^{b}+V_{\mu i}^{a}\left[D_{\mu}^{a b} \bar{\phi}_{i}^{b}+g \varepsilon^{a b}\left(\partial_{\mu} c+g \varepsilon^{c d} A_{\mu}^{c} c^{d}\right) \bar{\omega}_{i}^{b}\right]-\Omega_{\mu}^{a} D_{\mu}^{a b} c^{b}-g \varepsilon^{a b} \tau_{\mu}^{a} A_{\mu}^{b} c \\
& +\xi_{\mu}^{a}\left[g \varepsilon^{a b}\left(D_{\mu}^{b c} c^{c}\right) c-\frac{g^{2}}{2} \varepsilon^{a b} \varepsilon^{c d} A_{\mu}^{b} c^{c} c^{d}\right]-\Omega_{\mu}\left(\partial_{\mu} c+g \varepsilon^{a b} A_{\mu}^{a} c^{b}\right)+g \varepsilon^{a b} L^{a} c^{b} c+\frac{g}{2} \varepsilon^{a b} L c^{a} c^{b} \\
& +g \varepsilon^{a b} \lambda_{i}^{a} \phi_{i}^{b} c+\eta_{i}^{a}\left[g \varepsilon^{a b} \omega_{i}^{b} c+\frac{g^{2}}{2} \varepsilon^{a b} \varepsilon^{c d} \phi_{i}^{b} c^{c} c^{d}\right]+g \varepsilon^{a b} \rho_{i}^{a} \bar{\omega}_{i}^{b} c-\vartheta_{i}^{a}\left[g \varepsilon^{a b} \bar{\phi}_{i}^{b} c-\frac{g^{2}}{2} \varepsilon^{a b} \varepsilon^{c d} \bar{\omega}_{i}^{b} c^{c} c^{d}\right] \\
& \left.\quad+\chi\left(M_{\mu i}^{a} V_{\mu i}^{a}+U_{\mu i}^{a} N_{\mu i}^{a}\right)\right) \\
& +\frac{\alpha}{2} \int d^{4} x\left(b^{a} b^{a}-2 g \varepsilon^{a b} b^{a} \bar{c}^{b} c+g^{2} \bar{c}^{a} c^{a} \bar{c}^{b} c^{b}+g^{2}\left(\bar{\phi}_{i}^{a} \phi_{i}^{a}-\bar{\omega}_{i}^{a} \omega_{i}^{a}\right)\left(\bar{\phi}_{j}^{b} \phi_{j}^{b}-\bar{\omega}_{j}^{b} \omega_{j}^{b}\right)\right. \\
& \left.\quad-2 g^{2}\left(\bar{\phi}_{i}^{a} \phi_{i}^{a}-\bar{\omega}_{i}^{a} \omega_{i}^{a}\right) \bar{c}^{b} c^{b}+2 g^{2} \bar{\omega}_{i}^{a} \phi_{i}^{a} b^{b} c^{b}-2 g^{3} \bar{\omega}_{i}^{a} \phi_{i}^{a} \varepsilon^{b c} \bar{c}^{b} c^{c} c\right) .
\end{aligned}
$$

One should notice that the term quadratic in the sources $\left(M_{\mu i}^{a}, V_{\mu i}^{a}, U_{\mu i}^{a}, N_{\mu i}^{a}\right)$, i.e. $\chi\left(M_{\mu i}^{a} V_{\mu i}^{a}+\right.$ $U_{\mu i}^{a} N_{\mu i}^{a}$ ), in eq.(176) is of dimension four. As such, it is allowed by power counting and has to be added for renormalizability purposes. The parameter $\chi$ stands for a free coefficient.

Expression (76) represents our starting action.

\subsection{Ward identities}

It turns out that the classical action $\Sigma$, eq.(176), obeys the following set of Ward identities:

- the Slavnov-Taylor identity

$$
\mathcal{S}(\Sigma)=0
$$

with

$$
\begin{aligned}
\mathcal{S}(\Sigma) \equiv & \int d^{4} x\left(\left(\frac{\delta \Sigma}{\delta \Omega_{\mu}^{a}}+\frac{\delta \Sigma}{\delta \tau_{\mu}^{a}}\right) \frac{\delta \Sigma}{\delta A_{\mu}^{a}}+\frac{\delta \Sigma}{\delta \Omega_{\mu}} \frac{\delta \Sigma}{\delta A_{\mu}}+\frac{\delta \Sigma}{\delta L^{a}} \frac{\delta \Sigma}{\delta c^{a}}+\frac{\delta \Sigma}{\delta L} \frac{\delta \Sigma}{\delta c}+b^{a} \frac{\delta \Sigma}{\delta \bar{c}^{a}}+b \frac{\delta \Sigma}{\delta c}\right. \\
& \left.+\omega_{i}^{a} \frac{\delta \Sigma}{\delta \phi_{i}^{a}}+\bar{\phi}_{i}^{a} \frac{\delta \Sigma}{\delta \bar{\omega}_{i}^{a}}+N_{\mu i}^{a} \frac{\delta \Sigma}{\delta V_{\mu i}^{a}}-M_{\mu i}^{a} \frac{\delta \Sigma}{\delta U_{\mu i}^{a}}-\left(\Omega_{\mu}^{a}-\tau_{\mu}^{a}\right) \frac{\delta \Sigma}{\delta \xi_{\mu}^{a}}+\lambda_{i}^{a} \frac{\delta \Sigma}{\delta \eta_{i}^{a}}+\rho_{i}^{a} \frac{\delta \Sigma}{\delta \vartheta_{i}^{a}}\right),
\end{aligned}
$$


- the Ward identities corresponding to the $\delta_{i}$ and $\tilde{\delta}_{i}$ symmetries, eqs.(170), (711), i.e.

$$
\begin{aligned}
& \mathcal{W}_{i}(\Sigma) \equiv \int d^{4} x\left(\phi_{i}^{a} \frac{\delta \Sigma}{\delta \bar{c}^{a}}+c^{a} \frac{\delta \Sigma}{\delta \bar{\phi}_{i}^{a}}+V_{\mu i}^{a} \frac{\delta \Sigma}{\delta \Omega_{\mu}^{a}}-\vartheta_{i}^{a} \frac{\delta \Sigma}{\delta L^{a}}+\frac{\delta \Sigma}{\delta \lambda_{i}^{a}} \frac{\delta \Sigma}{\delta b^{a}}\right)=0, \\
& \widetilde{\mathcal{W}}_{i}(\Sigma) \equiv \int d^{4} x\left(\bar{\omega}_{i}^{a} \frac{\delta \Sigma}{\delta \bar{c}^{a}}-c^{a} \frac{\delta \Sigma}{\delta \omega_{i}^{a}}-U_{\mu i}^{a} \frac{\delta \Sigma}{\delta \Omega_{\mu}^{a}}+\eta_{i}^{a} \frac{\delta \Sigma}{\delta L^{a}}+\frac{\delta \Sigma}{\delta \rho_{i}^{a}} \frac{\delta \Sigma}{\delta b^{a}}\right)=0,
\end{aligned}
$$

- the $\mathcal{Q}_{i}(\Sigma)$ and $\widetilde{\mathcal{Q}}_{i}(\Sigma)$ identities obtained by commuting the Slavnov-Taylor identity, eq.(77), with the $\mathcal{W}_{i}(\Sigma)$ and $\widetilde{\mathcal{W}}_{i}(\Sigma)$ identities, eqs.(79), (80), namely

$$
\mathcal{Q}_{i}(\Sigma)=0,
$$

$\mathcal{Q}_{i}(\Sigma) \equiv \int d^{4} x\left(\left(\frac{\delta \Sigma}{\delta \lambda_{i}^{a}}+\omega_{i}^{a}\right) \frac{\delta \Sigma}{\delta \bar{c}^{a}}+\frac{\delta \Sigma}{\delta \eta_{i}^{a}} \frac{\delta \Sigma}{\delta b^{a}}+\left(\frac{\delta \Sigma}{\delta \bar{\phi}_{i}^{a}}-\rho_{i}^{a}\right) \frac{\delta \Sigma}{\delta L^{a}}+c^{a} \frac{\delta \Sigma}{\delta \bar{\omega}_{i}^{a}}-V_{\mu i}^{a} \frac{\delta \Sigma}{\delta \xi_{\mu}^{a}}+N_{\mu i}^{a} \frac{\delta \Sigma}{\delta \Omega_{\mu}^{a}}\right)$

and

$$
\begin{gathered}
\widetilde{\mathcal{Q}}_{i}(\Sigma)=0 \\
\widetilde{\mathcal{Q}}_{i}(\Sigma) \equiv \int d^{4} x\left(\left(\frac{\delta \Sigma}{\delta \rho_{i}^{a}}-\bar{\phi}_{i}^{a}\right) \frac{\delta \Sigma}{\delta \bar{c}^{a}}+\frac{\delta \Sigma}{\delta \vartheta_{i}^{a}} \frac{\delta \Sigma}{\delta b^{a}}+\left(\frac{\delta \Sigma}{\delta \omega_{i}^{a}}-\lambda_{i}^{a}\right) \frac{\delta \Sigma}{\delta L^{a}}-c^{a} \frac{\delta \Sigma}{\delta \phi_{i}^{a}}+U_{\mu i}^{a} \frac{\delta \Sigma}{\delta \xi_{\mu}^{a}}-M_{\mu i}^{a} \frac{\delta \Sigma}{\delta \Omega_{\mu}^{a}}\right)
\end{gathered}
$$

- the rigid $\mathcal{R}$-identities

$$
\begin{array}{r}
\mathcal{R}_{i j}^{(1)}(\Sigma) \equiv \int d^{4} x\left(\phi_{i}^{a} \frac{\delta \Sigma}{\delta \omega_{j}^{a}}-\bar{\omega}_{j}^{a} \frac{\delta \Sigma}{\delta \bar{\phi}_{i}^{a}}+V_{\mu i}^{a} \frac{\delta \Sigma}{\delta N_{\mu j}^{a}}+U_{\mu j}^{a} \frac{\delta \Sigma}{\delta M_{\mu i}^{a}}+\vartheta_{i}^{a} \frac{\delta \Sigma}{\delta \rho_{j}^{a}}-\eta_{j}^{a} \frac{\delta \Sigma}{\delta \lambda_{i}^{a}}\right)=0, \\
\mathcal{R}_{i j}^{(2)}(\Sigma) \equiv \int d^{4} x\left(\phi_{i}^{a} \frac{\delta \Sigma}{\delta \phi_{j}^{a}}-\bar{\phi}_{j}^{a} \frac{\delta \Sigma}{\delta \bar{\phi}_{i}^{a}}+\omega_{i}^{a} \frac{\delta \Sigma}{\delta \omega_{j}^{a}}-\bar{\omega}_{j}^{a} \frac{\delta \Sigma}{\delta \bar{\omega}_{i}^{a}}+V_{\mu i}^{a} \frac{\delta \Sigma}{\delta V_{\mu j}^{a}}-M_{\mu j}^{a} \frac{\delta \Sigma}{\delta M_{\mu i}^{a}}\right. \\
\left.+N_{\mu i}^{a} \frac{\delta \Sigma}{\delta N_{\mu j}^{a}}-U_{\mu j}^{a} \frac{\delta \Sigma}{\delta U_{\mu i}^{a}}+\vartheta_{i}^{a} \frac{\delta \Sigma}{\delta \vartheta_{j}^{a}}-\eta_{j}^{a} \frac{\delta \Sigma}{\delta \eta_{i}^{a}}+\rho_{i}^{a} \frac{\delta \Sigma}{\delta \rho_{j}^{a}}-\lambda_{j}^{a} \frac{\delta \Sigma}{\delta \lambda_{i}^{a}}\right)=0 \\
\mathcal{R}^{(3)}(\Sigma) \equiv \int d^{4} x\left(\bar{\omega}_{i}^{a} \frac{\delta \Sigma}{\delta \omega_{i}^{a}}-U_{\mu i}^{a} \frac{\delta \Sigma}{\delta N_{\mu i}^{a}}-\eta_{i}^{a} \frac{\delta \Sigma}{\delta \rho_{i}^{a}}\right)=0 \\
\mathcal{R}^{(4)}(\Sigma) \equiv \int d^{4} x\left(\bar{\omega}_{i}^{a} \frac{\delta \Sigma}{\delta \phi_{i}^{a}}-\bar{\phi}_{i}^{a} \frac{\delta \Sigma}{\delta \omega_{i}^{a}}-U_{\mu i}^{a} \frac{\delta \Sigma}{\delta V_{\mu i}^{a}}-M_{\mu i}^{a} \frac{\delta \Sigma}{\delta N_{\mu i}^{a}}-\eta_{i}^{a} \frac{\delta \Sigma}{\delta \vartheta_{i}^{a}}+\lambda_{i}^{a} \frac{\delta \Sigma}{\delta \rho_{i}^{a}}\right)=0,
\end{array}
$$


The trace of $\mathcal{R}_{i j}^{(2)}$, eq. (844), defines the $\mathcal{Q}_{f}$-charge of all fields and sources.

- the diagonal gauge-fixing condition and the antighost equation

$$
\begin{aligned}
& \frac{\delta \Sigma}{\delta b}=\partial_{\mu} A_{\mu}, \\
& \frac{\delta \Sigma}{\delta \bar{c}}+\partial_{\mu} \frac{\delta \Sigma}{\delta \Omega_{\mu}}=0 .
\end{aligned}
$$

- the $\mathcal{D}(\Sigma)$-Ward identity

$$
\mathcal{D}(\Sigma) \equiv \int d^{4} x\left(c^{a} \frac{\delta \Sigma}{\delta \bar{c}^{a}}+\frac{\delta \Sigma}{\delta L^{a}} \frac{\delta \Sigma}{\delta b^{a}}\right)=0 .
$$

- the local $U(1)$ Ward identity

$$
\mathcal{W}^{3}(\Sigma)=-\partial^{2} b
$$

with

$$
\begin{aligned}
\mathcal{W}^{3} \equiv & \partial_{\mu} \frac{\delta}{\delta A_{\mu}}+g \varepsilon^{a b}\left(A_{\mu}^{a} \frac{\delta}{\delta A_{\mu}^{b}}+b^{a} \frac{\delta}{\delta b^{b}}+c^{a} \frac{\delta}{\delta c^{b}}+\bar{c}^{a} \frac{\delta}{\delta \bar{c}^{b}}+\phi_{i}^{a} \frac{\delta}{\delta \phi_{i}^{b}}+\bar{\phi}_{i}^{a} \frac{\delta}{\delta \bar{\phi}_{i}^{b}}\right. \\
& +\omega_{i}^{a} \frac{\delta}{\delta \omega_{i}^{b}}+\bar{\omega}_{i}^{a} \frac{\delta}{\delta \bar{\omega}_{i}^{b}}+\Omega_{\mu}^{a} \frac{\delta}{\delta \Omega_{\mu}^{b}}+\tau_{\mu}^{a} \frac{\delta}{\delta \tau_{\mu}^{b}}+\xi_{\mu}^{a} \frac{\delta}{\delta \xi_{\mu}^{b}}+U_{\mu i}^{a} \frac{\delta}{\delta U_{\mu i}^{b}}+V_{\mu i}^{a} \frac{\delta}{\delta V_{\mu i}^{b}} \\
+ & \left.M_{\mu i}^{a} \frac{\delta}{\delta M_{\mu i}^{b}}+N_{\mu i}^{a} \frac{\delta}{\delta N_{\mu i}^{b}}+\eta_{i}^{a} \frac{\delta}{\delta \eta_{i}^{b}}+\vartheta_{i}^{a} \frac{\delta}{\delta \vartheta_{i}^{b}}+\lambda_{i}^{a} \frac{\delta}{\delta \lambda_{i}^{b}}+\rho_{i}^{a} \frac{\delta}{\delta \rho_{i}^{b}}+L^{a} \frac{\delta}{\delta L^{b}}\right) .
\end{aligned}
$$

Notice that the breaking term in the right hand side of eq.(901), i.e. $\partial^{2} b$, is linear in the quantum fields. It is thus a classical breaking, not affected by the quantum corrections [42].

\subsection{Useful commutation and anti-commutation relations}

For further use, let us give here the relevant commutation and anti-commutation relations between the nilpotent linearized Slavnov-Taylor operator $\mathcal{B}_{\Sigma}$,

$$
\begin{gathered}
\mathcal{B}_{\Sigma} \mathcal{B}_{\Sigma}=0, \\
\mathcal{B}_{\Sigma}=\int d^{4} x\left(\left(\frac{\delta \Sigma}{\delta \Omega_{\mu}^{a}}+\frac{\delta \Sigma}{\delta \tau_{\mu}^{a}}\right) \frac{\delta}{\delta A_{\mu}^{a}}+\frac{\delta \Sigma}{\delta A_{\mu}^{a}}\left(\frac{\delta}{\delta \Omega_{\mu}^{a}}+\frac{\delta}{\delta \tau_{\mu}^{a}}\right)+\frac{\delta \Sigma}{\delta \Omega_{\mu}} \frac{\delta}{\delta A_{\mu}}+\frac{\delta \Sigma}{\delta A_{\mu}} \frac{\delta}{\delta \Omega_{\mu}}\right. \\
+\frac{\delta \Sigma}{\delta L^{a}} \frac{\delta}{\delta c^{a}}+\frac{\delta \Sigma}{\delta c^{a}} \frac{\delta}{\delta L^{a}}+\frac{\delta \Sigma}{\delta L} \frac{\delta}{\delta c}+\frac{\delta \Sigma}{\delta c} \frac{\delta}{\delta L}+b^{a} \frac{\delta}{\delta \bar{c}^{a}}+b \frac{\delta}{\delta \bar{c}}+\omega_{i}^{a} \frac{\delta}{\delta \phi_{i}^{a}} \\
\left.+\bar{\phi}_{i}^{a} \frac{\delta}{\delta \bar{\omega}_{i}^{a}}+N_{\mu i}^{a} \frac{\delta}{\delta V_{\mu i}^{a}}-M_{\mu i}^{a} \frac{\delta}{\delta U_{\mu i}^{a}}-\left(\Omega_{\mu}^{a}-\tau_{\mu}^{a}\right) \frac{\delta}{\delta \xi_{\mu}^{a}}+\lambda_{i}^{a} \frac{\delta}{\delta \eta_{i}^{a}}+\rho_{i}^{a} \frac{\delta}{\delta \vartheta_{i}^{a}}\right),
\end{gathered}
$$


and the linearized operators $\mathcal{W}_{i}^{\Sigma}, \widetilde{\mathcal{W}}_{i}^{\Sigma}, \mathcal{Q}_{i}^{\Sigma}, \widetilde{\mathcal{Q}}_{i}^{\Sigma}, \mathcal{D}_{\Sigma}$ corresponding to the Ward identities (179), (80), (81), (82), (89), given by

$$
\begin{aligned}
& \mathcal{W}_{i}^{\Sigma}= \int d^{4} x\left(\phi_{i}^{a} \frac{\delta}{\delta \bar{c}^{a}}+c^{a} \frac{\delta}{\delta \bar{\phi}_{i}^{a}}+V_{\mu i}^{a} \frac{\delta}{\delta \Omega_{\mu}^{a}}-\vartheta_{i}^{a} \frac{\delta}{\delta L^{a}}+\frac{\delta \Sigma}{\delta \lambda_{i}^{a}} \frac{\delta}{\delta b^{a}}+\frac{\delta \Sigma}{\delta b^{a}} \frac{\delta}{\delta \lambda_{i}^{a}}\right), \\
& \widetilde{\mathcal{W}}_{i}^{\Sigma}=\int d^{4} x\left(\bar{\omega}_{i}^{a} \frac{\delta}{\delta \bar{c}^{a}}-c^{a} \frac{\delta}{\delta \omega_{i}^{a}}-U_{\mu i}^{a} \frac{\delta}{\delta \Omega_{\mu}^{a}}+\eta_{i}^{a} \frac{\delta}{\delta L^{a}}+\frac{\delta \Sigma}{\delta \rho_{i}^{a}} \frac{\delta}{\delta b^{a}}+\frac{\delta \Sigma}{\delta b^{a}} \frac{\delta}{\delta \rho_{i}^{a}}\right), \\
& \mathcal{Q}_{i}^{\Sigma}=\int d^{4} x\left(\left(\frac{\delta \Sigma}{\delta \lambda_{i}^{a}}+\omega_{i}^{a}\right) \frac{\delta}{\delta \bar{c}^{a}}-\frac{\delta \Sigma}{\delta \bar{c}^{a}} \frac{\delta}{\delta \lambda_{i}^{a}}+\frac{\delta \Sigma}{\delta \eta_{i}^{a}} \frac{\delta}{\delta b^{a}}+\frac{\delta \Sigma}{\delta b^{a}} \frac{\delta}{\delta \eta_{i}^{a}}+\left(\frac{\delta \Sigma}{\delta \bar{\phi}_{i}^{a}}-\rho_{i}^{a}\right) \frac{\delta}{\delta L^{a}}\right. \\
&\left.+\frac{\delta \Sigma}{\delta L^{a}} \frac{\delta}{\delta \bar{\phi}_{i}^{a}}+c^{a} \frac{\delta}{\delta \bar{\omega}_{i}^{a}}-V_{\mu i}^{a} \frac{\delta}{\delta \xi_{\mu}^{a}}+N_{\mu i}^{a} \frac{\delta}{\delta \Omega_{\mu}^{a}}\right), \\
& \widetilde{\mathcal{Q}}_{i}^{\Sigma}= \int d^{4} x\left(\left(\frac{\delta \Sigma}{\delta \rho_{i}^{a}}-\bar{\phi}_{i}^{a}\right) \frac{\delta}{\delta \bar{c}^{a}}+\frac{\delta \Sigma}{\delta \bar{c}^{a}} \frac{\delta}{\delta \rho_{i}^{a}}+\frac{\delta \Sigma}{\delta \vartheta_{i}^{a}} \frac{\delta}{\delta b^{a}}+\frac{\delta \Sigma}{\delta b^{a}} \frac{\delta}{\delta \vartheta_{i}^{a}}+\left(\frac{\delta \Sigma}{\delta \omega_{i}^{a}}-\lambda_{i}^{a}\right) \frac{\delta}{\delta L^{a}}\right. \\
&\left.+\frac{\delta \Sigma}{\delta L^{a}} \frac{\delta}{\delta \omega_{i}^{a}}-c^{a} \frac{\delta}{\delta \phi_{i}^{a}}+U_{\mu i}^{a} \frac{\delta}{\delta \xi_{\mu}^{a}}-M_{\mu i}^{a} \frac{\delta}{\delta \Omega_{\mu}^{a}}\right),
\end{aligned}
$$

and

$$
\mathcal{D}_{\Sigma}=\int d^{4} x\left(c^{a} \frac{\delta}{\delta \bar{c}^{a}}+\frac{\delta \Sigma}{\delta L^{a}} \frac{\delta}{\delta b^{a}}+\frac{\delta \Sigma}{\delta b^{a}} \frac{\delta}{\delta L^{a}}\right) .
$$

The commutation and anti-commutation relations of these linearized operators are found to be

$$
\begin{aligned}
\left\{\mathcal{W}_{i}^{\Sigma}, \mathcal{B}_{\Sigma}\right\} & =\mathcal{Q}_{i}^{\Sigma} \\
{\left[\widetilde{\mathcal{W}}_{i}^{\Sigma}, \mathcal{B}_{\Sigma}\right] } & =\widetilde{\mathcal{Q}}_{i}^{\Sigma} \\
{\left[\mathcal{D}_{\Sigma}, \mathcal{B}_{\Sigma}\right] } & =0 .
\end{aligned}
$$

Furthermore, we also have

$$
\begin{aligned}
\left\{\mathcal{R}_{i j}^{(1)}, \mathcal{B}_{\Sigma}\right\} & =\mathcal{R}_{i j}^{(2)} \\
{\left[\mathcal{R}^{(3)}, \mathcal{B}_{\Sigma}\right] } & =\mathcal{R}^{(4)} \\
{\left[\frac{\delta}{\delta b}, \mathcal{B}_{\Sigma}\right] } & =\frac{\delta}{\delta \bar{c}}+\partial_{\mu} \frac{\delta}{\delta \Omega_{\mu}} \\
{\left[\mathcal{W}^{3}, \mathcal{B}_{\Sigma}\right] } & =0
\end{aligned}
$$

\section{Algebraic characterization of the most general counter- term}

According to the algebraic renormalization [42, the most general local counterterm $\Sigma_{\mathrm{CT}}$ allowed by the Ward identities (77), (79), (80), (81), (82), (83), (84), (85), (86), (87), (88), (89), (90), is an integrated local polynomial in the fields and sources of dimension four, see Tables (2) and (3), which obeys the following set of constraints

$$
\mathcal{B}_{\Sigma} \Sigma_{\mathrm{CT}}=0
$$




\begin{tabular}{lcccccccc}
\hline & $A$ & $b$ & $\bar{c}$ & $c$ & $\phi$ & $\bar{\phi}$ & $\omega$ & $\bar{\omega}$ \\
\hline \hline dimension & 1 & 2 & 2 & 0 & 1 & 1 & 1 & 1 \\
gh number & 0 & 0 & -1 & 1 & 0 & 0 & 1 & -1 \\
$\mathcal{Q}_{f}$-charge & 0 & 0 & 0 & 0 & 1 & -1 & 1 & -1 \\
\hline
\end{tabular}

Table 2: Quantum numbers of the fields

\begin{tabular}{lcccccccccccc}
\hline & $\Omega$ & $\tau$ & $\xi$ & $L$ & $M$ & $N$ & $U$ & $V$ & $\eta$ & $\lambda$ & $\vartheta$ & $\rho$ \\
\hline \hline dimension & 3 & 3 & 3 & 4 & 2 & 2 & 2 & 2 & 3 & 3 & 3 & 3 \\
gh number & -1 & -1 & -2 & -2 & 0 & 1 & -1 & 0 & -2 & -1 & -1 & 0 \\
$\mathcal{Q}_{f}$-charge & 0 & 0 & 0 & 0 & -1 & 1 & -1 & 1 & -1 & -1 & 1 & 1 \\
\hline
\end{tabular}

Table 3: Quantum numbers of the sources

$$
\begin{gathered}
\mathcal{W}_{i}^{\Sigma} \Sigma_{\mathrm{CT}}=0, \\
\widetilde{\mathcal{W}}_{i}^{\Sigma} \Sigma_{\mathrm{CT}}=0, \\
\mathcal{Q}_{i}^{\Sigma} \Sigma_{\mathrm{CT}}=0, \\
\widetilde{\mathcal{Q}}_{i}^{\Sigma} \Sigma_{\mathrm{CT}}=0, \\
\mathcal{R}_{i j}^{(1)} \Sigma_{\mathrm{CT}}=0, \\
\mathcal{R}_{i j}^{(2)} \Sigma_{\mathrm{CT}}=0, \\
\mathcal{R}^{(3)} \Sigma_{\mathrm{CT}}=0, \\
\mathcal{R}^{(4)} \Sigma_{\mathrm{CT}}=0, \\
\mathcal{D}_{\Sigma} \Sigma_{\mathrm{CT}}=0, \\
\mathcal{W}^{3} \Sigma_{\mathrm{CT}}=0, \\
\frac{\delta \Sigma_{\mathrm{CT}}}{\delta b}=0, \\
\frac{\delta \Sigma_{\mathrm{CT}}}{\delta \bar{c}}+\partial_{\mu} \frac{\delta \Omega_{\mu}}{\delta \Sigma_{\mathrm{C}}}=0 .
\end{gathered}
$$

From eqs.(107) it follows that $\Sigma_{\mathrm{CT}}$ is independent of the diagonal Lagrange multiplier $b$, and that the diagonal antighost $\bar{c}$ enters only through the combination $\left(\Omega_{\mu}+\partial_{\mu} \bar{c}\right)$. Furthermore, from general results on the cohomology of gauge theories [42, 43], it turns out that the most general solution of the constraint (101) can be written as

$$
\Sigma_{\mathrm{CT}}=a_{0} S_{\mathrm{YM}}+\mathcal{B}_{\Sigma} \Delta^{(-1)}
$$

with $\Delta^{(-1)}$ being an integrated local polynomial with ghost number -1 , given by 


$$
\begin{aligned}
\Delta^{(-1)}= & \int d^{4} x\left(a_{1} \Omega_{\mu}^{a} A_{\mu}^{a}+a_{2} \tau_{\mu}^{a} A_{\mu}^{a}+a_{3} \xi_{\mu}^{a} g \varepsilon^{a b} A_{\mu}^{b} c+a_{4} \xi_{\mu}^{a} \partial_{\mu} c^{a}+a_{5} \xi_{\mu}^{a} g \varepsilon^{a b} A_{\mu} c^{b}+a_{6}\left(\partial_{\mu} \bar{c}^{a}\right) A_{\mu}^{a}\right. \\
& +a_{7}\left(\Omega_{\mu}+\partial_{\mu} \bar{c}\right) A_{\mu}+a_{8} c^{a} L^{a}+a_{9} c L+a_{10} \eta_{i}^{a} g \varepsilon^{a b} \phi_{i}^{b} c+a_{11} \eta_{i}^{a} \omega_{i}^{a}+a_{12} \vartheta_{i}^{a} g \varepsilon \varepsilon^{a b} \bar{\omega}_{i}^{b} c \\
& +a_{13} \vartheta_{i}^{a} \bar{\phi}_{i}^{a}+a_{14} \lambda_{i}^{a} \phi_{i}^{a}+a_{15} \rho_{i}^{a} \bar{\omega}_{i}^{a}+a_{16} U_{\mu i}^{a} \partial_{\mu} \phi_{i}^{a}+a_{17} U_{\mu i}^{a} g \varepsilon^{a b} A_{\mu} \phi_{i}^{b}+a_{18} V_{\mu i}^{a} \partial_{\mu} \bar{\omega}_{i}^{a} \\
& +a_{19} V_{\mu i}^{a} g \varepsilon^{a b} A_{\mu} \bar{\omega}_{i}^{b}+a_{20} \bar{c}^{a} b^{a}+a_{21} g \varepsilon^{a b} \bar{c}^{a} \bar{c}^{b} c+a_{22} g \varepsilon^{a b} \bar{c}^{a} A_{\mu} A_{\mu}^{b}+a_{23} \bar{\omega}_{i}^{a} \phi_{i}^{a} \bar{\phi}_{j}^{b} \phi_{j}^{b} \\
& +a_{24} \bar{\omega}_{i}^{a} \phi_{i}^{a} \bar{\omega}_{j}^{b} \omega_{j}^{b}+a_{25} \bar{\omega}_{i}^{a} \phi_{i}^{b} \bar{\phi}_{j}^{a} \phi_{j}^{b}+a_{26} \bar{\omega}_{i}^{a} \phi_{i}^{b} \bar{\omega}_{j}^{a} \omega_{j}^{b}+a_{27} \bar{\omega}_{i}^{a} \phi_{i}^{b} \bar{\phi}_{j}^{b} \phi_{j}^{a}+a_{28} \bar{\omega}_{i}^{a} \phi_{i}^{b} \bar{\omega}_{j}^{b} \omega_{j}^{a} \\
& +a_{29} \bar{\omega}_{i}^{a} \phi_{j}^{a} \bar{\phi}_{i}^{b} \phi_{j}^{b}+a_{30} \bar{\omega}_{i}^{a} \phi_{j}^{b} \bar{\phi}_{i}^{a} \phi_{j}^{b}+a_{31} \bar{\omega}_{i}^{a} \phi_{j}^{a} \bar{\omega}_{i}^{b} \omega_{j}^{b}+a_{32} \bar{\omega}_{i}^{a} \phi_{i}^{a} \bar{c}^{b} c^{b}+a_{33} \bar{\omega}_{i}^{a} \phi_{i}^{b} \bar{c}^{a} c^{b} \\
& +a_{34} \bar{\omega}_{i}^{a} \phi_{i}^{b} \bar{c}^{b} c^{a}+a_{35} \bar{\omega}_{i}^{a} \phi_{i}^{a} A_{\mu} A_{\mu}+a_{36} \bar{\omega}_{i}^{a} \phi_{i}^{a} A_{\mu}^{b} A_{\mu}^{b}+a_{37} \bar{\omega}_{i}^{a} \phi_{i}^{b} A_{\mu}^{a} A_{\mu}^{b}+a_{38} \bar{\omega}_{i}^{a} \partial^{2} \phi_{i}^{a} \\
& \left.+a_{39} \bar{\omega}_{i}^{a} g \varepsilon^{a b} A_{\mu} \partial_{\mu} \phi_{i}^{b}+a_{40} \bar{\omega}_{i}^{a} g \varepsilon^{a b}\left(\partial_{\mu} A_{\mu}\right) \phi_{i}^{b}+a_{41} \chi U_{\mu i}^{a} V_{\mu i}^{a}\right)
\end{aligned}
$$

where the coefficients $a_{i}, i=0, \ldots, 41$ are free dimensionless parameters. Notice also that in the derivation of expression (109) use has been made of the fact that the action $\Sigma$, and thus $\Sigma_{\mathrm{CT}}$, are left invariant by the following discrete symmetry

$$
\begin{aligned}
\Psi^{1} & \rightarrow \Psi^{1}, \\
\Psi^{2} & \rightarrow-\Psi^{2}, \\
\Psi^{\text {diag }} & \rightarrow-\Psi^{\text {diag }},
\end{aligned}
$$

where $\Psi^{a}, a=1,2$, and $\Psi^{\text {diag }}$ stand, respectively, for all off-diagonal and diagonal fields and external sources. As one can easily recognize, this symmetry plays the role of the charge conjugation [11].

After a rather long calculation, from the constraints (102), (103), (104), (105), (106), one finds that

$$
\begin{aligned}
a_{7} & =a_{10}=a_{11}=a_{12}=a_{13}=a_{14}=a_{15}=0, \\
a_{25} & =a_{26}=a_{27}=a_{28}=a_{29}=a_{30}=a_{31}=0, \\
a_{33} & =a_{34}=0,
\end{aligned}
$$

while

$$
\begin{aligned}
a_{3} & =a_{1} \\
a_{5} & =-a_{4} \\
a_{22} & =a_{6} \\
a_{16} & =-a_{17}=-a_{18}=a_{19}=a_{1}-a_{4}+a_{8} \\
a_{21} & =-a_{20}, \\
a_{23} & =-a_{24}=g^{2} a_{20}-\alpha g^{2} a_{8}, \\
a_{32} & =-2 g^{2} a_{20}+\alpha g^{2} a_{8}, \\
a_{38} & =-a_{40}=-\frac{a_{39}}{2}=-\frac{a_{35}}{g^{2}}=\frac{a_{36}}{g^{2}}=-\frac{a_{37}}{g^{2}}=a_{6}+a_{8}
\end{aligned}
$$


Making use of the relation

$$
\tau_{\mu}^{a} A_{\mu}^{a}=\Omega_{\mu}^{a} A_{\mu}^{a}+\xi_{\mu}^{a}\left(D_{\mu}^{a b} c^{b}+g \varepsilon^{a b} A_{\mu}^{b} c\right)+\mathcal{B}_{\Sigma}\left(\xi_{\mu}^{a} A_{\mu}^{a}\right)
$$

and remembering that $\mathcal{B}_{\Sigma}^{2}=0$, we obtain

$$
\begin{aligned}
\Delta^{(-1)}= & \int d^{4} x\left(\left(a_{1}+a_{2}\right)\left[\Omega_{\mu}^{a} A_{\mu}^{a}+g \varepsilon^{a b} \xi_{\mu}^{a} A_{\mu}^{b} c\right]+\left(a_{2}+a_{4}\right) \xi_{\mu}^{a} D_{\mu}^{a b} c^{b}\right. \\
& +\left(a_{1}-a_{4}+a_{8}\right)\left[U_{\mu i}^{a} D_{\mu}^{a b} \phi_{i}^{b}-V_{\mu i}^{a} D_{\mu}^{a b} \bar{\omega}_{i}^{a}\right]-\left(a_{6}+a_{8}\right) \bar{\omega}_{i}^{a} \mathcal{M}^{a b} \phi_{i}^{a} \\
& -a_{6} \bar{c}^{a} D_{\mu}^{a b} A_{\mu}^{b}+a_{8} L^{a} c^{a}+a_{9} L c+a_{41} \chi U_{\mu i}^{a} V_{\mu i}^{a}+a_{20}\left(\bar{c}^{a} b^{a}-g \varepsilon^{a b} \bar{c}^{a} \bar{c}^{b} c\right) \\
& \left.-\left(\alpha a_{8}-a_{20}\right) g^{2} \bar{\omega}_{i}^{a} \phi_{i}^{a}\left(\bar{\phi}_{j}^{b} \phi_{j}^{b}-\bar{\omega}_{j}^{b} \omega_{j}^{b}\right)+\left(\alpha a_{8}-2 a_{20}\right) g^{2} \bar{\omega}_{i}^{a} \phi_{i}^{a} \bar{c}^{b} c^{b}\right)
\end{aligned}
$$

Finally, by renaming the coefficients as follows

$$
\begin{aligned}
a_{1}+a_{2} & \rightarrow a_{1}, \\
a_{2}+a_{4} & \rightarrow-a_{2}, \\
a_{8} & \rightarrow a_{3}, \\
a_{6} & \rightarrow a_{4}, \\
a_{9} & \rightarrow a_{5}, \\
a_{41} & \rightarrow a_{6}, \\
a_{20} & \rightarrow \frac{\alpha}{2} a_{7}
\end{aligned}
$$


for the most general local allowed counterterm $\Sigma_{\mathrm{CT}}$, we obtain

$$
\begin{aligned}
& \Sigma_{\mathrm{CT}}=\int d^{4} x\left(( a _ { 0 } + 2 a _ { 1 } ) \left[\frac{1}{2}\left(\partial_{\mu} A_{\nu}^{a}\right)\left(\partial_{\mu} A_{\nu}^{a}-\partial_{\nu} A_{\mu}^{a}\right)-g \varepsilon^{a b}\left(\partial_{\mu} A_{\nu}^{a}\right)\left(A_{\mu} A_{\nu}^{b}-A_{\nu} A_{\mu}^{b}\right)\right.\right. \\
& \left.+g \varepsilon^{a b}\left(\partial_{\mu} A_{\nu}^{a}\right) A_{\mu}^{a} A_{\mu}^{b}+\frac{g^{2}}{2}\left(A_{\mu} A_{\mu} A_{\nu}^{a} A_{\nu}^{a}+A_{\mu} A_{\nu} A_{\mu}^{a} A_{\nu}^{a}\right)\right]+\frac{a_{0}}{2}\left(\partial_{\mu} A_{\nu}\right)\left(\partial_{\mu} A_{\nu}-\partial_{\nu} A_{\mu}\right) \\
& +\left(a_{0}+4 a_{1}\right) \frac{g^{2}}{4} A_{\mu}^{a} A_{\mu}^{a} A_{\nu}^{b} A_{\nu}^{b}+\left(a_{1}-a_{4}\right) b^{a} D_{\mu}^{a b} A_{\mu}^{b} \\
& -\left(a_{3}+a_{4}\right)\left[\bar{c}^{a} \partial^{2} c^{a}-\bar{c}^{a} g \varepsilon^{a b}\left(\partial_{\mu} A_{\mu}\right) c^{b}-2 \bar{c}^{a} g \varepsilon^{a b} A_{\mu} \partial_{\mu} c^{b}-g^{2} \bar{c}^{a} c^{a} A_{\mu} A_{\mu}\right] \\
& +\left(a_{3}+a_{4}\right)\left[\bar{\phi}_{i}^{a} \partial^{2} \phi_{i}^{a}-\bar{\phi}_{i}^{a} g \varepsilon^{a b}\left(\partial_{\mu} A_{\mu}\right) \phi_{i}^{b}-2 \bar{\phi}_{i}^{a} g \varepsilon^{a b} A_{\mu} \partial_{\mu} \phi_{i}^{b}-g^{2} \bar{\phi}_{i}^{a} \phi_{i}^{a} A_{\mu} A_{\mu}\right] \\
& -\left(a_{3}+a_{4}\right)\left[\bar{\omega}_{i}^{a} \partial^{2} \omega_{i}^{a}-\bar{\omega}_{i}^{a} g \varepsilon^{a b}\left(\partial_{\mu} A_{\mu}\right) \omega_{i}^{b}-2 \bar{\omega}_{i}^{a} g \varepsilon^{a b} A_{\mu} \partial_{\mu} \omega_{i}^{b}-g^{2} \bar{\omega}_{i}^{a} \omega_{i}^{a} A_{\mu} A_{\mu}\right] \\
& +\left(2 a_{1}-a_{3}-a_{4}\right) g^{2} \varepsilon^{a c} \varepsilon^{b d}\left(\bar{c}^{a} c^{b}-\bar{\phi}_{i}^{a} \phi_{i}^{b}+\bar{\omega}_{i}^{a} \omega_{i}^{b}\right) A_{\mu}^{c} A_{\mu}^{d} \\
& +\left(a_{1}-a_{4}-a_{5}\right) g \varepsilon^{a b} \bar{c}^{a} c D_{\mu}^{b c} A_{\mu}^{c}-\left(a_{1}-a_{3}\right)\left(\Omega_{\mu}+\partial_{\mu} \bar{c}\right) g \varepsilon^{a b} A_{\mu}^{a} c^{b} \\
& +\left(a_{1}-2 a_{3}-a_{4}\right)\left[2 g^{2} \varepsilon^{a b} \varepsilon^{c d} \bar{\omega}_{i}^{a} A_{\mu}^{c} c^{d} \partial_{\mu} \phi_{i}^{b}+g^{2} \varepsilon^{a b} \varepsilon^{c d} \bar{\omega}_{i}^{a} \partial_{\mu}\left(A_{\mu}^{c} c^{d}\right) \phi_{i}^{b}\right. \\
& \left.+2 g^{3} \varepsilon^{b c} \bar{\omega}_{i}^{a} \phi_{i}^{a} A_{\mu} A_{\mu}^{b} c^{c}-g^{2}\left(\varepsilon^{a c} \varepsilon^{b d}+\varepsilon^{a d} \varepsilon^{b c}\right) \bar{\omega}_{i}^{a} A_{\mu}^{d}\left(D_{\mu}^{c e} c^{e}\right) \phi_{i}^{b}\right] \\
& +\left(2 a_{1}-a_{3}-a_{4}-a_{5}\right) g^{3}\left(\delta^{a e} \varepsilon^{b d}+\delta^{b e} \varepsilon^{a d}\right) \bar{\omega}_{i}^{a} A_{\mu}^{d} A_{\mu}^{e} \phi_{i}^{b} c \\
& -\left(a_{2}+2 a_{3}\right) g^{2} \varepsilon^{a b} \varepsilon^{c d}\left(U_{\mu i}^{a} \phi_{i}^{b}+V_{\mu i}^{a} \bar{\omega}_{i}^{b}\right) A_{\mu}^{c} c^{d}+\left(a_{1}+a_{2}+a_{3}\right) \Omega_{\mu}^{a} D_{\mu}^{a b} c^{b} \\
& -a_{2} \tau_{\mu}^{a} D_{\mu}^{a b} c^{b}+a_{5} g \varepsilon^{a b} \tau_{\mu}^{a} A_{\mu}^{b} c-\left(a_{1}+a_{2}+a_{3}+a_{5}\right) g \varepsilon^{a b} \xi_{\mu}^{a}\left(D_{\mu}^{a b} c^{c}\right) c \\
& +\left(a_{2}+2 a_{3}\right) \frac{g^{2}}{2} \varepsilon^{a b} \varepsilon^{c d} \xi_{\mu}^{a} A_{\mu}^{b} c^{c} c^{d}-a_{5} g \varepsilon^{a b} L^{a} c^{b} c-\left(2 a_{3}-a_{5}\right) \frac{g^{2}}{2} \varepsilon^{a b} L c^{a} c^{b} \\
& +a_{5}\left(\Omega_{\mu}+\partial_{\mu} \bar{c}\right) \partial_{\mu} c-a_{3} g^{2} \varepsilon^{a b} \varepsilon^{c d}\left(\eta_{i}^{a} \phi_{i}^{b}+\vartheta_{i}^{a} \bar{\omega}_{i}^{b}\right) c^{c} c^{d}-a_{5} g \varepsilon^{a b}\left(\lambda_{i}^{a} \phi_{i}^{b}+\eta_{i}^{a} \omega_{i}^{b}\right. \\
& \left.+\rho_{i}^{a} \bar{\omega}_{i}^{b}-\vartheta_{i}^{a} \bar{\phi}_{i}^{b}\right) c-\left(a_{3}+a_{4}+a_{5}\right)\left[2 g \varepsilon^{a b} \bar{\omega}_{i}^{a}\left(\partial_{\mu} c\right) \partial_{\mu} \phi_{i}^{b}+g \varepsilon^{a b} \bar{\omega}_{i}^{a}\left(\partial^{2} c\right) \phi_{i}^{b}\right. \\
& \left.+2 g^{2} \bar{\omega}_{i}^{a} \phi_{i}^{a} A_{\mu} \partial_{\mu} c\right]+\left(a_{1}+a_{2}+a_{3}+a_{5}\right) g \varepsilon^{a b}\left(\partial_{\mu} c\right)\left(U_{\mu i}^{a} \phi_{i}^{b}-V_{\mu i}^{a} \bar{\omega}_{i}^{b}\right) \\
& -\left(a_{1}+a_{2}+a_{3}\right)\left(M_{\mu i}^{a} D_{\mu}^{a b} \phi_{i}^{b}+U_{\mu i}^{a} D_{\mu}^{a b} \omega_{i}^{b}+V_{\mu i}^{a} D_{\mu}^{a b} \bar{\phi}_{i}^{b}+N_{\mu i}^{a} D_{\mu}^{a b} \bar{\omega}_{i}^{b}\right) \\
& \left.-a_{6} \chi\left(M_{\mu i}^{a} V_{\mu i}^{a}+U_{\mu i}^{a} N_{\mu i}^{a}\right)\right) \\
& +\frac{\alpha}{2} \int d^{4} x\left(a_{7} b^{a} b^{a}-2\left(a_{7}-a_{5}\right) g \varepsilon^{a b} b^{a} \bar{c}^{b} c+g^{2}\left(a_{7}-2 a_{3}\right) \bar{c}^{a} c^{a} \bar{c}^{b} c^{b}\right. \\
& +g^{2}\left(a_{7}-2 a_{3}\right)\left(\bar{\phi}_{i}^{a} \phi_{i}^{a}-\bar{\omega}_{i}^{a} \omega_{i}^{a}\right)\left(\bar{\phi}_{j}^{b} \phi_{j}^{b}-\bar{\omega}_{j}^{b} \omega_{j}^{b}\right)-2 g^{2}\left(a_{7}-2 a_{3}\right)\left(\bar{\phi}_{i}^{a} \phi_{i}^{a}-\bar{\omega}_{i}^{a} \omega_{i}^{a}\right) \bar{c}^{b} c^{b} \\
& \left.+2 g^{2}\left(a_{7}-2 a_{3}\right) \bar{\omega}_{i}^{a} \phi_{i}^{a} b^{b} c^{b}-2 g^{3}\left(a_{7}-2 a_{3}-a_{5}\right) \bar{\omega}_{i}^{a} \phi_{i}^{a} \varepsilon^{b c} \bar{c}^{b} c^{c} c\right) \text {. }
\end{aligned}
$$

\subsection{Stability of the classical action and renormalization factors}

After the characterization of the most general local counterterm $\Sigma_{\mathrm{CT}}$, eq.(116), compatible with all Ward identities, we still have to check that it can be reabsorbed through a multiplicative redefinition of the fields, sources and parameters of the starting action $\Sigma$, according to

$$
\Sigma\left(\Psi_{0}, \psi_{0}, J_{0}, \Omega_{0}, \tau_{0}, \zeta_{0}\right)=\Sigma(\Psi, \psi, J, \Omega, \tau, \zeta)+\epsilon \Sigma_{\mathrm{CT}}(\Psi, \psi, J, \Omega, \tau, \zeta)+O\left(\epsilon^{2}\right)
$$

where

$$
\Psi_{0}=\widetilde{Z}_{\Psi}^{1 / 2} \Psi
$$


denotes the off-diagonal fields, $\Psi \equiv\left(A_{\mu}^{a}, b^{a}, c^{a}, \bar{c}^{a}\right)$, while

$$
\psi_{0}=Z_{\psi}^{1 / 2} \psi,
$$

stands for the fields, $\psi \equiv\left(A_{\mu}, b, c, \bar{c}, \phi_{i}^{a}, \bar{\phi}_{i}^{a}, \omega_{i}^{a}, \bar{\omega}_{i}^{a}\right)$.

Also $J$ and $\zeta$

$$
\begin{aligned}
& J_{0}=Z_{J} J, \\
& \zeta_{0}=Z_{\zeta} \zeta,
\end{aligned}
$$

denote the external sources, $J \equiv\left(\xi_{\mu}^{a}, L^{a}, L, \Omega_{\mu}, U_{\mu i}^{a}, V_{\mu i}^{a}, M_{\mu i}^{a}, N_{\mu i}^{a}, \eta_{i}^{a}, \lambda_{i}^{a}, \vartheta_{i}^{a}, \rho_{i}^{a}\right)$, and the parameters $\zeta \equiv(g, \chi, \alpha)$, respectively.

Moreover, recalling that the sources $\Omega_{\mu}^{a}$ and $\tau_{\mu}^{a}$ are coupled to composite operators, $\mathcal{O}_{1}=-D_{\mu}^{a b} c^{b}$, $\mathcal{O}_{2}=-g \varepsilon^{a b} A_{\mu}^{b} c$, displaying the same quantum numbers, see eqs.(65),(67), they renormalize as

$$
\left(\begin{array}{c}
\Omega_{0 \mu}^{a} \\
\tau_{0 \mu}^{a}
\end{array}\right)=Z_{\Omega \tau}\left(\begin{array}{c}
\Omega_{\mu}^{a} \\
\tau_{\mu}^{a}
\end{array}\right)
$$

where the matrix

$$
Z_{\Omega \tau}=1+\epsilon\left(\begin{array}{cc}
z_{\Omega} & z_{\Omega \tau} \\
0 & z_{\tau}
\end{array}\right)
$$

allows for the mixing at the quantum level between the operators $\mathcal{O}_{1}, \mathcal{O}_{2}$. By direct inspection of $\Sigma_{\mathrm{CT}}$, the renormalization factors are easily found to be

$$
\begin{aligned}
\widetilde{Z}_{b} & =Z_{g}^{2} Z_{\bar{c}}^{-1} \widetilde{Z}_{c}, \\
Z_{b} & =Z_{g}^{2} \\
Z_{\Omega} & =Z_{\bar{c}}^{1 / 2} \\
Z_{\bar{\omega}} & =Z_{g}^{-2} Z_{\bar{c}} \widetilde{Z}_{c}, \\
Z_{\omega} & =Z_{g}^{2} Z_{\bar{c}}^{-1} \widetilde{Z}_{c} \\
Z_{\phi} & =Z_{\bar{\phi}}=\widetilde{Z}_{c}, \\
Z_{U} & =Z_{g}^{-1} Z_{\bar{c}}^{1 / 2} Z_{V} \\
Z_{N} & =Z_{g} Z_{\bar{c}}^{-1 / 2} Z_{V} \\
Z_{M} & =Z_{V} \\
Z_{\eta} & =Z_{g}^{-2} Z_{\bar{c}} \widetilde{Z}_{c}^{-1 / 2}, \\
Z_{\lambda} & =Z_{\vartheta}=Z_{g}^{-1} Z_{\bar{c}}^{1 / 2} \widetilde{Z}_{c}^{-1 / 2}, \\
Z_{\rho} & =\widetilde{Z}_{c}^{-1 / 2} \\
Z_{\xi} & =Z_{V} \\
Z_{L^{a}} & =Z_{g}^{-1} Z_{\bar{c}}^{1 / 2} \widetilde{Z}_{c}^{-1 / 2}, \\
Z_{L} & =Z_{g}^{-1} Z_{\bar{c}}^{1 / 2} Z_{c}^{-1 / 2}, \\
Z_{A} & =Z_{g}^{-2},
\end{aligned}
$$


with

$$
\begin{aligned}
\widetilde{Z}_{A} & =1+\epsilon\left(a_{0}+2 a_{1}\right) \\
Z_{g} & =1-\epsilon \frac{a_{0}}{2} \\
\widetilde{Z}_{c} & =\widetilde{Z}_{\bar{c}}=1-\epsilon\left(a_{3}+a_{4}\right) \\
Z_{c} & =1+\epsilon\left(a_{3}-a_{4}-2 a_{5}\right) \\
Z_{\bar{c}} & =1-\epsilon\left(a_{3}-a_{4}\right) \\
Z_{V} & =1+\epsilon\left(-a_{1}-a_{2}-\frac{a_{3}}{2}+\frac{a_{4}}{2}\right) \\
Z_{\chi} & =1+\epsilon\left(2 a_{1}+2 a_{2}+a_{3}-a_{4}-a_{6}\right) \\
Z_{\alpha} & =1+\epsilon\left(a_{7}+a_{0}+2 a_{4}\right)
\end{aligned}
$$

Finally

$$
Z_{\Omega \tau}=\left(\begin{array}{cc}
Z_{V} & Z_{g}^{-1} Z_{\bar{c}}^{1 / 2} Z_{V}^{-1} \widetilde{Z}_{A}^{-1 / 2}-1 \\
0 & Z_{g}^{-1} Z_{\bar{c}}^{1 / 2} \widetilde{Z}_{A}^{-1 / 2}
\end{array}\right) .
$$

Equations (123) , (124), (125) show that the counterterm $\Sigma_{\mathrm{CT}}$ can be reabsorbed by means of a redefinition of the fields, sources and parameters of the starting action $\Sigma$, establishing thus the multiplicative renormalizability of the theory. Let us end this section by noting that the renormalization factors $\left(Z_{\phi}, Z_{\bar{\phi}}, Z_{\bar{\omega}}, Z_{\omega}\right)$ of the auxiliary localizing fields $\left(\phi_{i}^{a}, \bar{\phi}_{i}^{a}, \omega_{i}^{a}, \bar{\omega}_{i}^{a}\right)$ are not independent quantities, being expressed in terms of the renormalization factors of the gauge coupling constant and of the Faddeev-Popov ghosts. Again, this feature stems from the Ward identities (79), (80).

We also notice that the renormalization factor $Z_{g}$ of the gauge coupling constant $g$ can be obtained directly from the renormalization factor $Z_{A}$ of the diagonal component of the gauge field $A_{\mu}$. Thus, the nonrenormalization theorem of the maximal Abelian gauge [11, 12]

$$
Z_{A} Z_{g}^{2}=1
$$

remains valid in the presence of the Gribov horizon. This important property is a direct consequence of the local $U(1)$ Ward identity (90).

\section{Introduction of a generalized dimension two local oper- ator}

As remarked in the Introduction, the inclusion of the horizon term, eq.(8), does not prevent us from defining a local composite dimension two operator $\mathcal{O}_{A^{2}}$

$$
\mathcal{O}_{A^{2}} \equiv \frac{1}{2} A_{\mu}^{a} A_{\mu}^{a}+\alpha\left(\bar{c}^{a} c^{a}-\bar{\phi}_{i}^{a} \phi_{i}^{a}+\bar{\omega}_{i}^{a} \omega_{i}^{a}\right)
$$

which generalizes the gluon operator $\left(\frac{1}{2} A_{\mu}^{a} A_{\mu}^{a}+\alpha \bar{c}^{a} c^{a}\right)$ already considered in the maximal Abelian gauge [4], and proven to be renormalizable to all orders [12]. From the equation of motion of the Lagrange multiplier $b^{a}$

$$
\frac{\delta \Sigma}{\delta b^{a}}=D_{\mu}^{a b} A_{\mu}^{b}+\alpha\left(b^{a}-g \varepsilon^{a b} \bar{c}^{b} c+g^{2} \bar{\omega}_{i}^{b} \phi_{i}^{b} c^{a}\right)
$$


it follows that the integrated operator $\int d^{4} x \mathcal{O}_{A^{2}}$ enjoys the property of being $B R S T$ invariant on-shell, namely

$$
s \int d^{4} x \mathcal{O}_{A^{2}}=\int d^{4} x\left(-\left(D_{\mu}^{a b} c^{b}\right) A_{\mu}^{a}+\alpha b^{a} c^{a}-\alpha g \bar{c}^{a} \varepsilon^{a b} c^{b} c\right)=\int d^{4} x c^{a} \frac{\delta \Sigma}{\delta b^{a}} .
$$

As proven in the Landau gauge [45, 46, 22] in the case of the operator $A_{\mu}^{A} A_{\mu}^{A}, A=1, \ldots, N^{2}-1$, eq.(129) implies that the local composite operator $\mathcal{O}_{A^{2}}$ is multiplicatively renormalizable. This can be established by introducing $\mathcal{O}_{A^{2}}$ in the starting action $\Sigma$, and by repeating the algebraic analysis done in the previous section. More precisely, we introduce the operator $\mathcal{O}_{A^{2}}$ by means of a $B R S T$ doublet of external sources $(J, \Lambda)$

$$
s \Lambda=J, \quad s J=0,
$$

so that

$$
\begin{aligned}
\widetilde{\Sigma} & =\Sigma+s \int d^{4} x\left(\Lambda \mathcal{O}_{A^{2}}+\frac{\varrho}{2} \Lambda J\right) \\
& =\Sigma+\int d^{4} x\left(J \mathcal{O}_{A^{2}}+\Lambda\left(A_{\mu}^{a} D_{\mu}^{a b} c^{b}-\alpha b^{a} c^{a}+\alpha g \bar{c}^{a} \varepsilon^{a b} c^{b} c\right)+\frac{\varrho}{2} J^{2}\right) .
\end{aligned}
$$

The parameter $\varrho$ is a free parameter, needed in order to account for the ultraviolet divergences of the correlation function $\left\langle\left(A_{\mu}^{a}(x) A_{\mu}^{a}(x)\right)\left(A_{\nu}^{b}(y) A_{\nu}^{b}(y)\right)\right\rangle[12$.

The extended action $\widetilde{\Sigma}$ is easily seen to obey the following Slavnov-Taylor identity

$$
\mathcal{S}(\widetilde{\Sigma})=0
$$

with

$$
\begin{aligned}
\mathcal{S}(\widetilde{\Sigma})= & \int d^{4} x\left(\left(\frac{\delta \widetilde{\Sigma}}{\delta \Omega_{\mu}^{a}}+\frac{\delta \widetilde{\Sigma}}{\delta \tau_{\mu}^{a}}\right) \frac{\delta \widetilde{\Sigma}}{\delta A_{\mu}^{a}}+\frac{\delta \widetilde{\Sigma}}{\delta \Omega_{\mu}} \frac{\delta \widetilde{\Sigma}}{\delta A_{\mu}}+\frac{\delta \widetilde{\Sigma}}{\delta L^{a}} \frac{\delta \widetilde{\Sigma}}{\delta c^{a}}+\frac{\delta \widetilde{\Sigma}}{\delta L} \frac{\delta \widetilde{\Sigma}}{\delta c}\right. \\
& +b^{a} \frac{\delta \widetilde{\Sigma}}{\delta \bar{c}^{a}}+b \frac{\delta \widetilde{\Sigma}}{\delta c}+\omega_{i}^{a} \frac{\delta \widetilde{\Sigma}}{\delta \phi_{i}^{a}}+\bar{\phi}_{i}^{a} \frac{\delta \widetilde{\Sigma}}{\delta \bar{\omega}_{i}^{a}}+N_{\mu i}^{a} \frac{\delta \widetilde{\Sigma}}{\delta V_{\mu i}^{a}}-M_{\mu i}^{a} \frac{\delta \widetilde{\Sigma}}{\delta U_{\mu i}^{a}} \\
& \left.-\left(\Omega_{\mu}^{a}-\tau_{\mu}^{a}\right) \frac{\delta \widetilde{\Sigma}}{\delta \xi_{\mu}^{a}}+\lambda_{i}^{a} \frac{\delta \widetilde{\Sigma}}{\delta \eta_{i}^{a}}+\rho_{i}^{a} \frac{\delta \widetilde{\Sigma}}{\delta \vartheta_{i}^{a}}+J \frac{\delta \widetilde{\Sigma}}{\delta \Lambda}\right) .
\end{aligned}
$$

All other Ward identities, eqs.(79-90), turn out to hold, remaining unmodified by the introduction of the sources $(J, \Lambda)$. Moreover, there is an additional Ward identity

$$
\mathcal{U}(\widetilde{\Sigma})=\int d^{4} x\left(\frac{\delta \widetilde{\Sigma}}{\delta \Lambda}+\bar{c}^{a} \frac{\delta \widetilde{\Sigma}}{\delta b^{a}}\right)=0
$$

expressing the fact that the integrated operator $\int d^{4} x \mathcal{O}_{A^{2}}$ is $B R S T$ invariant on-shell.

By repeating the same analysis as before, for the most general local counterterm we obtain

$$
\widetilde{\Sigma}_{\mathrm{CT}}=a_{0} S_{\mathrm{YM}}+\mathcal{B}_{\widetilde{\Sigma}} \widetilde{\Delta}^{(-1)},
$$


with

$$
\begin{aligned}
\widetilde{\Delta}^{(-1)}= & \int d^{4} x\left(a_{1}\left[\Omega_{\mu}^{a} A_{\mu}^{a}+\xi_{\mu}^{a} g \varepsilon^{a b} A_{\mu}^{b} c\right]-a_{2} \xi_{\mu}^{a} D_{\mu}^{a b} c^{b}+a_{3} L^{a} c^{a}-a_{4} \bar{c}^{a} D_{\mu}^{a b} A_{\mu}^{b}\right. \\
& +a_{5} L c+a_{6} \chi U_{\mu i}^{a} V_{\mu i}^{a}+\left(a_{1}+a_{2}+a_{3}\right)\left[U_{\mu i}^{a} D_{\mu}^{a b} \phi_{i}^{b}-V_{\mu i}^{a} D_{\mu}^{a b} \bar{\omega}_{i}^{b}\right]-\left(a_{3}+a_{4}\right) \bar{\omega}_{i}^{a} \mathcal{M}^{a b} \phi_{i}^{b} \\
& +\frac{\alpha}{2} a_{7}\left(\bar{c}^{a} b^{a}-g \varepsilon^{a b} \bar{c}^{a} \bar{c}^{b} c\right)+\frac{\alpha}{2}\left(a_{7}-2 a_{3}\right) g^{2} \bar{\omega}_{i}^{a} \phi_{i}^{a}\left(\bar{\phi}_{j}^{b} \phi_{j}^{b}-\bar{\omega}_{j}^{b} \omega_{j}^{b}\right)-\alpha\left(a_{7}-a_{3}\right) g^{2} \bar{\omega}_{i}^{a} \phi_{i}^{a} \bar{c}^{b} c^{b} \\
& \left.+\Lambda\left(\frac{1}{2}\left(a_{3}-a_{4}\right) A_{\mu}^{a} A_{\mu}^{a}+\alpha\left(a_{7}+a_{3}\right) \bar{c}^{a} c^{a}-\alpha a_{7}\left(\bar{\phi}_{j}^{b} \phi_{j}^{b}-\bar{\omega}_{j}^{b} \omega_{j}^{b}\right)+a_{8} \frac{\varrho}{2} J\right)\right)
\end{aligned}
$$

In particular, for the renormalization of the sources $J$ and $\Lambda$, and of the parameter $\varrho$ one finds

$$
\begin{aligned}
J_{0} & =Z_{J} J=Z_{g}^{2} Z_{\bar{c}}^{-1} J, \\
\Lambda_{0} & =Z_{\Lambda} \Lambda=Z_{g} Z_{\bar{c}}^{-1 / 2} \Lambda, \\
\varrho_{0} & =Z_{\varrho} \varrho=\left(1+\epsilon\left(2 a_{0}-2 a_{3}+2 a_{4}+a_{8}\right)\right) \varrho .
\end{aligned}
$$

We see thus that the renormalization of the source $J$, and thus of the composite operator coupled to it, i.e. $\mathcal{O}_{A^{2}}$, can be expressed in terms of the renormalization factors of the gauge coupling constant $g$ and of the diagonal antighost $\bar{c}$, meaning that the anomalous dimension of $\mathcal{O}_{A^{2}}$ is not an independent parameter of the theory. Again, this result is in complete analogy with the case of the Landau gauge, where the corresponding operator $A_{\mu}^{A} A_{\mu}^{A}$ is multiplicatively renormalizable ${ }^{7}$ [22] in the presence of Zwanziger's horizon function (31).

\section{Conclusion}

In this work we have pursued the study of the maximal Abelian gauge by taking into account the restriction of the domain of integration in the Faddeev-Popov quantization formula to the Gribov region $\mathcal{C}_{0}$. Such a restriction is needed due to the existence of Gribov copies. The Gribov approximation, previously introduced in [1], has been improved through the introduction of the nonlocal horizon function, eq.(8). As in the case of the Landau gauge [19, 20], the horizon term of the maximal Abelian gauge can be cast in local form with the help of additional auxiliary fields. The resulting local action $\Sigma$, eq.(176), turns out to be multiplicatively renormalizable to all orders. This is the main result of the present article. It could open new perspectives, motivating further analytic studies of the maximal Abelian gauge which might provide a better comparison with lattice numerical results [15, 16]. A partial list of the topics worth to be analysed can be summarized as follows:

- The possibility of having at our disposal a local and renormalizable action incorporating the effects of the Gribov horizon enables us to work out the higher order quantum corrections affecting both the diagonal and off-diagonal gluon propagator. We expect that the incorporation of these quantum corrections will provide a more direct and reliable comparison with the lattice data. As an example, we mention the possibility of investigating by analytical methods the behavior of the longitudinal off-diagonal component of the gluon propagator, which we were unable to discuss within Gribov's quadratic approximation [1]. Lattice results [16] have pointed out that this component is nonvanishing. We remark, however, that,

\footnotetext{
${ }^{7}$ Here too, the anomalous dimension of $A_{\mu}^{A} A_{\mu}^{A}$ is not an independent parameter of the theory [22].
} 
from the analytic point of view, it vanishes at the tree level. Nevertheless, it might arise at the quantum level due to the contribution of the diagonal and off-diagonal transverse components of the gluon propagator in the loop integrals of higher order Feynman diagrams. As these transverse components carry nonperturbative information, embodied in the Gribov parameter $\gamma$ and in the dynamical gluon mass $m$, a nonvanishing longitudinal component might show up at the quantum level. Certainly, the renormalizability of the starting action $\Sigma$ is a crucial ingredient here.

- A second issue to be investigated is the infrared behavior of the off-diagonal ghost propagator $\left\langle\bar{c}^{a}(k) c^{b}(-k)\right\rangle$, which can be decomposed into symmetric and antisymmetric part with respect to the off-diagonal indices $a, b=1,2$. As already mentioned, the symmetric part of the ghost propagator, i.e. $\sum_{a, b} \delta^{a b}\left\langle\bar{c}^{a}(k) c^{b}(-k)\right\rangle$, has been found to be enhanced in the infrared region, in the Gribov approximation [1], as expressed by eq.(22). A more detailed study of the behavior of the symmetric component of the ghost propagator would provide an important check in order to establish whether this infrared enhancement will be left unmodified by the higher order corrections.

Also, the study of the antisymmetric part of the ghost propagator, i.e. $\varepsilon^{a b}\left\langle\bar{c}^{a}(k) c^{b}(-k)\right\rangle$, would provide information about the phenomenon of the ghost condensation in the presence of the Gribov horizon. The possibility of the formation of a ghost condensate $\left\langle\varepsilon^{a b} \bar{c}^{a}(x) c^{b}(x)\right\rangle$ in the maximal Abelian gauge, proposed in [47, has been investigated by several authors [48, 49, 50]. This phenomenon has been shown to occur also in the Landau gauge [51, 52, 53], where a few lattice results are available [54, 55. Here, the ghost condensation has been established by constructing the effective potential for the operator $\varepsilon^{a b} \bar{c}^{a}(x) c^{b}(x)$. The existence of a nontrivial minimum for the effective potential resulted in a nonvanishing condensate $\left\langle\varepsilon^{a b} \bar{c}^{a} c^{b}\right\rangle$ [51, 52, 53. It could be interesting to see how the phenomenon of the ghost condensation would be modified by the presence of the Gribov horizon, a feature which can be now faced. This might result in a better comparison with the available lattice results ${ }^{8}$ on the ghost condensation, which are done by considering always gauge configurations which lie within the Gribov region. The construction of the effective potential for the ghost operator $\varepsilon^{a b} \bar{c}^{a}(x) c^{b}(x)$ in the presence of the Gribov horizon is under investigation. Both Landau and maximal Abelian gauge will be covered.

- The multiplicative renormalizability of the local composite operator $\mathcal{O}_{A^{2}}$, eq.(127), opens the possibility to perform a study of the dimension two gluon condensate $\left\langle\mathcal{O}_{A^{2}}\right\rangle$ when the restriction to the Gribov region $\mathcal{C}_{0}$ is taken into account. This might improve our understanding of the dynamical mass generation for off-diagonal gluons, a feature relevant for the dual superconductivity picture of color confinement.

- Finally, we would like to call attention to the nonrenormalization theorem

$$
Z_{A} Z_{g}^{2}=1
$$

which still holds in the presence of the horizon. This result, stemming from the local $U(1)$ Ward identity (90), could motivate further studies of the maximal Abelian gauge from both

${ }^{8}$ We are indebted to A. Cucchieri and T. Mendes for providing us their preliminary results about the numerical studies on the lattice of the ghost condensation in the maximal Abelian gauge. 
Schwinger-Dyson approach and lattice numerical simulations. Concerning the potential applications to the Schwinger-Dyson equations, we remark that eq.(138) could be employed in order to obtain a useful truncation scheme. Also, it would be interesting to look at relation (138) from the lattice point of view, as it could allow to study the infrared behavior of the running coupling constant by analysing the form factor of the pure diagonal gluon propagator.

\section{Acknowledgments}

The Conselho Nacional de Desenvolvimento Científico e Tecnológico (CNPq-Brazil), the Faperj, Fundação de Amparo à Pesquisa do Estado do Rio de Janeiro, the SR2-UERJ and the Coordenação de Aperfeiçoamento de Pessoal de Nível Superior (CAPES) are gratefully acknowledged for financial support.

\section{References.}

[1] M. A. L. Capri, V. E. R. Lemes, R. F. Sobreiro, S. P. Sorella and R. Thibes, Phys. Rev. D 72 (2005) 085021 arXiv:hep-th/0507052.

[2] G. 't Hooft, Nucl. Phys. B 190 (1981) 455.

[3] A. S. Kronfeld, G. Schierholz and U. J. Wiese, Nucl. Phys. B 293 (1987) 461.

[4] A. S. Kronfeld, M. L. Laursen, G. Schierholz and U. J. Wiese, Phys. Lett. B 198 (1987) 516.

[5] Y. Nambu, Phys. Rev. D10 (1974) 4262;

G. 't Hooft, High Energy Physics EPS Int. Conference, Palermo 1975, ed. A. Zichichi;

S. Mandelstam, Phys. Rept. 23 (1976) 245.

[6] Z. F. Ezawa and A. Iwazaki, Phys. Rev. D 25 (1982) 2681.

[7] T. Suzuki and I. Yotsuyanagi, Phys. Rev. D 42 (1990) 4257.

[8] T. Suzuki, S. Hioki, S. Kitahara, S. Kiura, Y. Matsubara, O. Miyamura and S. Ohno, Nucl. Phys. Proc. Suppl. 26 (1992) 441.

[9] S. Hioki, S. Kitahara, S. Kiura, Y. Matsubara, O. Miyamura, S. Ohno and T. Suzuki, Phys. Lett. B 272 (1991) 326 [Erratum-ibid. B 281 (1992) 416].

[10] H. Min, T. Lee and P. Y. Pac, Phys. Rev. D 32, 440 (1985).

[11] A. R. Fazio, V. E. R. Lemes, M. S. Sarandy and S. P. Sorella, Phys. Rev. D 64, 085003 (2001) arXiv:hep-th/0105060.

[12] D. Dudal, J. A. Gracey, V. E. R. Lemes, M. S. Sarandy, R. F. Sobreiro, S. P. Sorella and H. Verschelde, Phys. Rev. D 70, 114038 (2004) arXiv:hep-th/0406132.

[13] J. A. Gracey, JHEP 0504, 012 (2005) arXiv:hep-th/0504051. 
[14] L. Baulieu and D. Zwanziger, Nucl. Phys. B 548, 527 (1999) arXiv:hep-th/9807024.

[15] K. Amemiya and H. Suganuma, Phys. Rev. D 60 (1999) 114509.

[16] V. G. Bornyakov, M. N. Chernodub, F. V. Gubarev, S. M. Morozov and M. I. Polikarpov, Phys. Lett. B 559 (2003) 214.

[17] V. N. Gribov, Nucl. Phys. B 139 (1978) 1.

[18] F. Bruckmann, T. Heinzl, A. Wipf and T. Tok, Nucl. Phys. B 584, 589 (2000) arXiv:hep-th/0001175.

[19] D. Zwanziger, Nucl. Phys. B 323, 513 (1989).

[20] D. Zwanziger, Nucl. Phys. B 399, 477 (1993).

[21] N. Maggiore and M. Schaden, Phys. Rev. D 50, 6616 (1994) arXiv:hep-th/9310111.

[22] D. Dudal, R. F. Sobreiro, S. P. Sorella and H. Verschelde, Phys. Rev. D 72, 014016 (2005) arXiv:hep-th/0502183.

[23] J. A. Gracey, Phys. Lett. B 632, 282 (2006) arXiv:hep-ph/0510151.

[24] J. A. Gracey, JHEP 0605, 052 (2006) arXiv:hep-ph/0605077.

[25] D. B. Leinweber, J. I. Skullerud, A. G. Williams and C. Parrinello [UKQCD Collaboration], Phys. Rev. D 60, 094507 (1999) [Erratum-ibid. D 61, 079901 (2000)] arXiv:hep-lat/9811027.

[26] D. R. Bonnet, P. O. Bowman, D. B. Leinweber, A. G. Williams and J. M. Zanotti, Phys. Rev. D 64 (2001) 034501 arXiv:hep-lat/0101013.

[27] K. Langfeld, H. Reinhardt and J. Gattnar, Nucl. Phys. B 621, 131 (2002) arXiv:hep-ph/0107141.

[28] A. Cucchieri, T. Mendes and A. R. Taurines, Phys. Rev. D 67 (2003) 091502 Phys. Rev. D 67 (2003) 091502 arXiv:hep-lat/0302022.

[29] J. C. R. Bloch, A. Cucchieri, K. Langfeld and T. Mendes, Nucl. Phys. B 687 (2004) 76 arXiv:hep-lat/0312036.

[30] S. Furui and H. Nakajima, Phys. Rev. D 69 (2004) 074505 arXiv:hep-lat/0305010.

[31] P. J. Silva and O. Oliveira, Nucl. Phys. B 690, 177 (2004) arXiv:hep-lat/0403026.

[32] A. Cucchieri, T. Mendes and A. R. Taurines, Phys. Rev. D 71, 051902 (2005) arXiv:hep-lat/0406020.

[33] I. L. Bogolubsky, G. Burgio, M. Muller-Preussker and V. K. Mitrjushkin, Phys. Rev. D 74, 034503 (2006) arXiv:hep-lat/0511056.

[34] L. von Smekal, R. Alkofer and A. Hauck, Phys. Rev. Lett. 79 (1997) 3591 arXiv:hep-ph/9705242. 
[35] L. von Smekal, A. Hauck and R. Alkofer, Annals Phys. 267, 1 (1998) [Erratum-ibid. 269, 182 (1998)] arXiv:hep-ph/9707327.

[36] D. Atkinson and J. C. R. Bloch, Phys. Rev. D 58 (1998) arXiv:hep-ph/9712459.

[37] R. Alkofer and L. von Smekal, Phys. Rept. 353 (2001) 281 arXiv:hep-ph/0007355.

[38] P. Watson and R. Alkofer, Phys. Rev. Lett. 86 (2001) 5239 arXiv:hep-ph/0102332.

[39] M. A. L. Capri, D. Dudal, J. A. Gracey, V. E. R. Lemes, R. F. Sobreiro, S. P. Sorella and H. Verschelde, Phys. Rev. D 72, 105016 (2005) arXiv:hep-th/0510240.

[40] M. A. L. Capri, D. Dudal, J. A. Gracey, V. E. R. Lemes, R. F. Sobreiro, S. P. Sorella and H. Verschelde, Phys. Rev. D 74, 045008 (2006) arXiv:hep-th/0605288.

[41] G. Barnich and M. Henneaux, Phys. Lett. B 311, 123 (1993) arXiv:hep-th/9304057.

[42] O. Piguet and S. P. Sorella, "Algebraic renormalization: Perturbative renormalization, symmetries and anomalies", Lect. Notes Phys. M28, 1 (1995).

[43] G. Barnich, F. Brandt and M. Henneaux, Phys. Rept. 338, 439 (2000) arXiv:hep-th/0002245.

[44] K. I. Kondo, T. Murakami, T. Shinohara and T. Imai, Phys. Rev. D 65, 085034 (2002) arXiv:hep-th/0111256.

[45] H. Verschelde, K. Knecht, K. Van Acoleyen and M. Vanderkelen, Phys. Lett. B 516, 307 (2001) arXiv:hep-th/0105018.

[46] D. Dudal, H. Verschelde and S. P. Sorella, Phys. Lett. B 555, 126 (2003) arXiv:hep-th/0212182.

[47] M. Schaden, "Mass generation in continuum SU(2) gauge theory in covariant Abelian gauges", arXiv:hep-th/9909011.

[48] K. I. Kondo, Phys. Lett. B 514, 335 (2001) arXiv:hep-th/0105299.

[49] V. E. R. Lemes, M. S. Sarandy and S. P. Sorella, J. Phys. A 36, 7211 (2003) arXiv:hep-th/0206251.

[50] D. Dudal and H. Verschelde, J. Phys. A 36, 8507 (2003) arXiv:hep-th/0209025.

[51] V. E. R. Lemes, M. S. Sarandy and S. P. Sorella, Annals Phys. 308, 1 (2003) arXiv:hep-th/0210077.

[52] D. Dudal et al., JHEP 0306, 003 (2003) arXiv:hep-th/0305020.

[53] M. A. L. Capri, D. Dudal, J. A. Gracey, V. E. R. Lemes, R. F. Sobreiro, S. P. Sorella and H. Verschelde, Phys. Rev. D 73, 014001 (2006) arXiv:hep-th/0508216.

[54] A. Cucchieri, T. Mendes and A. Mihara, Phys. Rev. D 72, 094505 (2005) arXiv:hep-lat/0508028.

[55] S. Furui and H. Nakajima, Phys. Rev. D 73, 094506 (2006) arXiv:hep-lat/0602027. 